\title{
Aberrant cell migration contributes to defective airway epithelial repair in childhood wheeze
}

Thomas losifidis, $, 1,2,3$ Erika N. Sutanto, ${ }^{3}$ Alysia G. Buckley, ${ }^{4}$ Laura Coleman,,${ }^{1,3}$ Erin E. Gill, ${ }^{5}$ Amy H. Lee, ${ }^{5}$ Kak-Ming Ling, ${ }^{1,3}$ Jessica Hillas, ${ }^{3}$ Kevin Looi, ${ }^{3}$ Luke W. Garratt, ${ }^{3}$ Kelly M. Martinovich,,${ }^{1,3}$ Nicole C. Shaw, ${ }^{1,3}$ Samuel T. Montgomery, ${ }^{1,3}$ Elizabeth Kicic-Starcevich, ${ }^{3}$ Yuliya V. Karpievitch, ${ }^{3}$ Peter Le Souëf, ${ }^{1,3}$ Ingrid A. Laing, ${ }^{1,3}$ Shyan Vijayasekaran, ${ }^{1}$ Francis J. Lannigan, ${ }^{6}$ Paul J. Rigby, ${ }^{4}$ Robert E.W. Hancock, ${ }^{5,7}$ Darryl A. Knight, ${ }^{8,9,10}$ Stephen M. Stick, ${ }^{1,2,3,11}$ Anthony Kicic, ${ }^{1,2,3,11,12}$ Western Australian Epithelial Research Program (WAERP), ${ }^{13}$ and Australian Respiratory Epithelium Consortium (AusREC) ${ }^{13}$

'Division of Pediatrics and 2Centre for Cell Therapy and Regenerative Medicine, School of Medicine, University of Western Australia, Nedlands, Western Australia, Australia. ${ }^{3}$ Telethon Kids Institute Respiratory Research Centre, Perth, Western Australia, Australia. ${ }^{4}$ Centre of Microscopy, Characterisation and Analysis, University of Western Australia, Nedlands, Western Australia, Australia. ${ }^{5}$ Center for Microbial Diseases Research, University of British Columbia, Vancouver, British Columbia, Canada. ${ }^{6}$ School of Medicine, Notre Dame University, Fremantle, Western Australia, Australia. 'Wellcome Trust Sanger Institute, Wellcome Trust Genome Campus, Hinxton, Cambridge, United Kingdom. ${ }^{8}$ School of Biomedical Sciences and Pharmacy, University of Newcastle, Callaghan, New South Wales, Australia. ${ }^{9}$ Priority Research Centre for Healthy Lungs, Hunter Medical Research Institute, Newcastle, New South Wales, Australia. ${ }^{10}$ Department of Anesthesiology, Pharmacology and Therapeutics, University of British Columbia, Vancouver, Canada. "Department of Respiratory and Sleep Medicine, Perth Children's Hospital, Nedlands, Western Australia, Australia. ${ }^{12}$ School of Public Health, Curtin University, Bentley, Western Australia, Australia. ${ }^{13}$ See Supplemental Acknowledgments for consortium details.

Abnormal wound repair has been observed in the airway epithelium of patients with chronic respiratory diseases, including asthma. Therapies focusing on repairing vulnerable airways, particularly in early life, present a potentially novel treatment strategy. We report defective lower airway epithelial cell repair to strongly associate with common pre-school-aged and school-aged wheezing phenotypes, characterized by aberrant migration patterns and reduced integrin $\alpha 5 \beta 1$ expression. Next generation sequencing identified the PI3K/Akt pathway as the top upstream transcriptional regulator of integrin $\alpha 5 \beta 1$, where Akt activation enhanced repair and integrin $\alpha 5 \beta 1$ expression in primary cultures from children with wheeze. Conversely, inhibition of PI3K/Akt signaling in primary cultures from children without wheeze reduced $\alpha 5 \beta 1$ expression and attenuated repair. Importantly, the FDA-approved drug celecoxib - and its non-COX2-inhibiting analogue, dimethyl-celecoxib - stimulated the PI3K/Akt-integrin $\alpha 531$ axis and restored airway epithelial repair in cells from children with wheeze. When compared with published clinical data sets, the identified transcriptomic signature was also associated with viral-induced wheeze exacerbations highlighting the clinical potential of such therapy. Collectively, these results identify airway epithelial restitution via targeting the PI3K-integrin $\alpha 5 \beta 1$ axis as a potentially novel therapeutic avenue for childhood wheeze and asthma. We propose that the next step in the therapeutic development process should be a proof-of-concept clinical trial, since relevant animal models to test the crucial underlying premise are unavailable.

AU2019/050493)

Copyright: (ㄷ) 2020, American Society for Clinical Investigation.

Submitted: September 9, 2019

Accepted: March 4, 2020

Published: March 24, 2020

Reference information: /CI Insight. 2020;5(7):e133125.

https://doi.org/10.1172/jci.

insight.133125.

\section{Introduction}

Approximately 300 million individuals suffer from asthma globally (1), and it remains the commonest chronic respiratory disorder in children. The pathobiology of asthma is complex and poorly understood (2-4). While current therapies ameliorate symptoms, none affect the long-term trajectory of lung function decline (5-7). Despite the significantly high global prevalence of asthma, and the magnitude of research 
investment, there are few new therapies that aim to target specific disease mechanisms. Thus, there is an unmet need for new therapeutic avenues to be explored.

The airway epithelium is considered to be an important contributor to asthma development, possibly through intrinsic abnormalities that include dysregulated wound repair capacity and loss of barrier integrity (8-15). Unresolved airway epithelial restitution following insults, such as viral infection, have been associated with asthma exacerbations $(16,17)$ and drive chronic airway remodeling $(18-21)$. These features are evident in young children with wheeze, not necessarily subsequent to but rather in parallel with airway inflammation $(11,15,22-27)$, highlighting the potential for early interventions that target the epithelium to modify the recurrence of respiratory wheeze symptoms and disease trajectory.

In the airway, normal repair following injury is characterized by spreading and migration into a wound of leading edge cells that surround the wound site. This is followed by basal cell proliferation and differentiation into various epithelial cell subtypes. Migration alone can close small wounds, whereas repair of large areas requires proliferation and migration. We previously demonstrated that, although primary airway epithelial cells (pAEC) from children with asthma are hyperproliferative, they are ineffective at repairing wounds in vitro (11), in part due to deficient production of the extracellular matrix (ECM) protein fibronectin (FN) (27). With the intent of intervening in early life to prevent irreversible airway damage and persistence of symptoms, we recruited children with recurrent wheeze, as well as healthy controls without wheeze, to test the hypothesis that dysregulated airway epithelial cell repair was also characteristic of this cohort. Furthermore, knowing that airway epithelial cell migration is facilitated via the binding of membrane-bound integrins on their cell surface to FN and other ECM proteins (28-31), we also hypothesized that aberrant migration and dysregulation of integrin-related pathways contributes to deficient epithelial repair observed. Thus, we focused on the pattern and regulation of migration of leading edge cells following in vitro wounding.

In this study, we show that the defective airway epithelial cell repair phenotype associates with wheeze in pre-school-aged children and physician diagnosis of asthma. Abnormal leading edge cell migration impairs repair following injury in pAEC cultures from children with wheeze associated with reduced expression of integrin $\alpha 5 \beta 1$. Transcriptomic analysis of the leading edge migrating cells identified a number of upstream regulators of integrin expression and migration, of which blunted PI3K/Akt signaling resulted in reduced expression of integrin $\alpha 5 \beta 1$ and, as a consequence, pAEC migration and repair following wounding. Importantly, we showed that the PI3K/Akt-integrin $\alpha 5 \beta 1$ axis is therapeutically targetable with the US Food and Drug Administration-approved (FDA-approved) drug celecoxib and its experimental analogue, dimethyl-celecoxib. Furthermore, dysregulation of the PI3K/Akt-integrin $\alpha 5 \beta 1$ axis was also present in independent cohort data sets that associated with wheeze recurrence. Considering that its safety profile is known and it is currently prescribed to young children with juvenile arthritis, our findings warrant further preclinical assessment and initial trial of celecoxib in children with recurrent wheeze to improve symptom resolution and reduce length of stays in hospital via its enhancement of airway epithelial repair.

\section{Results}

Defective airway epithelial cell repair phenotype associates with respiratory wheeze in children. Previous observations by our group identified a defective repair phenotype in pAEC to associate with physician diagnosis of asthma in school-aged children $(11,27,32)$. This study assessed in vitro wound repair in pAEC cultures from 119 children with a median age 5.4 (IQR 3.3-8.2) years (Table 1). Defective pAEC repair was determined to associate strongly with respiratory wheeze in all children (Table 1). Specifically, defective pAEC repair associated with respiratory wheeze in pre-school-aged children ( $\leq 5$ years old) and school-aged children (>5 years old; Supplemental Table 1; supplemental material available online with this article; https:// doi.org/10.1172/jci.insight.133125DS1). Furthermore, the proportion of school-aged children ( $>5$ years old) with physician diagnosis of asthma and complete pAEC repair was significantly lower than their agematched nonasthmatic counterparts (Fisher exact test $P=1.223 \times 10^{-9}$; Supplemental Table 2), corroborating our previously published observations $(11,27,32)$.

Consistent with our previous findings $(11,27,32)$, pAEC from children without any respiratory conditions demonstrated a rapid repair response that was completed by 72 hours after wounding $(P$ $<0.050$, Figure 1A, Supplemental Video 1). In contrast, pAEC from children with wheeze displayed significantly compromised wound repair capacity and failed to fully repair over the duration of the experiment $(P<0.050$, Figure 1B, Supplemental Video 2$)$. As such, this study aimed to investigate the mechanisms regulating defective pAEC repair in children with respiratory wheeze. 
Table 1. Defective airway epithelial cell repair associates with childhood respiratory wheeze

\begin{tabular}{|c|c|c|c|c|c|}
\hline & Number & Estimate & Standard error & Z score & $\boldsymbol{P}$ value $(>|\mathrm{z}|)$ \\
\hline Defective pAEC repair & 49 & $\mathrm{~N} / \mathrm{A}$ & $\mathrm{N} / \mathrm{A}$ & N/A & $\mathrm{N} / \mathrm{A}$ \\
\hline Age at sampling, years ${ }^{A}$ & $5.4(3.3-8.2)$ & -0.098 & 0.076 & -1.287 & 0.198 \\
\hline Wheeze & 46 & -2.487 & 0.620 & -4.011 & $6.050 \times 10^{-5 B}$ \\
\hline
\end{tabular}

PAEC, primary airway epithelial cell. ${ }^{A}$ Median (IQR); ${ }^{\mathrm{B} P}<0.050$.

Aberrant cell migration contributes to defective repair in airway epithelial cells from children with wheeze. When the migration component of repair was assessed, leading edge cells from children without wheeze migrated consistently toward the center of the wound (Figure 1C, Supplemental Video 1). However, leading edge cells from children with wheeze had a highly variable trajectory distribution, lacking consistent directionality, with some cells migrating backward into the leading edge (Figure 1D, Supplemental Video 2). The significant reduction in distance migrated $(P<0.050$, Figure $1 \mathrm{E}$ and Supplemental Figure $1 \mathrm{~A})$, velocity $(P$ $<0.050$, Figure $1 \mathrm{~F}$ and Supplemental Figure 1B), directionality $(P<0.050$, Figure $1 \mathrm{G}$ and Supplemental Figure 1C), and overall forward motion $(P<0.050$, Figure $1 \mathrm{H}$ and Supplemental Figure $1 \mathrm{D})$ contributed to the failure of pAEC from children with wheeze to fully repair.

Integrin $\alpha 5 \beta 1$ regulates leading edge cell migration in the airway epithelium. Previous investigations by our group identified that reduced FN synthesis and release contributed to the defective repair of pAEC from children with asthma (27). Furthermore, addition of exogenous FN did not completely restore wound repair (27), suggesting that additional mechanisms contribute to the defective wound repair. We speculated that the expression of FN-binding integrins is also dysregulated from preasthmatics or recurrent wheezers and contributes to the defective repair that characterizes epithelial cells from this cohort.

All of the mRNA from FN-binding integrin subunits (i.e., $\alpha \mathrm{V}, \alpha 3, \alpha 5, \beta 1, \beta 3, \beta 6$ ) was expressed by pAEC from children without wheeze ex vivo, with ITGA5 and ITGB1 being the most abundantly expressed (Supplemental Figure 2, A-F). These were the only $\alpha$ and $\beta$ integrin subunits that were significantly decreased in pAEC of children with wheeze (ITGA5, 2.2-fold, $P<0.050$; ITGB1, 1.6-fold, $P<0.050$; Supplemental Figure 2, C and D). Similar to expression ex vivo, protein expression of both integrin subunits $\alpha 5$ and $\beta 1$ was significantly decreased in cultured pAEC from children with wheeze $(\alpha 5,2.3$-fold, $P<0.050$; $\beta 1,2.2$-fold, $P<0.050$; Figure 2, A and B).

In pAEC from children without wheeze, confocal imaging demonstrated strong staining intensity for integrin $\alpha 5$ along the first row of leading edge cells, following wounding, whereas staining intensity was progressively weaker in cells distal to the wound (Figure 2C). Expression of integrin $\beta 1$ was similar to $\alpha 5$ in cells along the leading edge but was almost undetectable in sites distal to the wound (Figure 2D). In contrast, integrin $\alpha 5$ and $\beta 1$ (Figure 2, F-H) protein staining of leading edge cells from children with wheeze was lower than that of their nonwheezing counterparts (Figure 2, C-E).

To confirm the role of integrins during airway epithelial repair, functional blocking experiments were performed in pAEC from children without wheeze. Since integrin $\alpha 5$ only forms a functional heterodimer with integrin $\beta 1$, and since $\beta 1$ can induce intracellular signaling to regulate cell migration, in these experiments, integrin $\beta 1$ was blocked with anti- $\beta 1$ integrin antibody. This resulted in the inhibition of wound closure of pAEC from children without wheeze in a concentration-dependent manner (Figure 2I, Supplemental Figure 3A) and dysregulated migration patterns (Figure 2, J and K; Supplemental Figure 3, B-F).

Altered transcriptional response to wounding and upstream regulators of integrin $\alpha 5 \beta 1$ expression in childhood wheeze. In order to determine major pathways with the capacity to influence migration and repair, we undertook whole genome transcriptional profiling of leading edge cells 24 hours following scratch wounding. Samples from children with wheeze displayed an altered transcriptional profile, as they formed distinct clusters from their nonwheezing counterparts (Figure 3A). A total of 1154 genes were differentially expressed (DE; fold change, $|1.5|$; Benjamini-Hochberg-adjusted $P<0.050$; Figure 3B), with 664 upregulated (Supplemental Table 3) and 490 downregulated genes (Supplemental Table 4), between leading edge pAEC from children with and without wheeze. These DE genes corresponded 
A
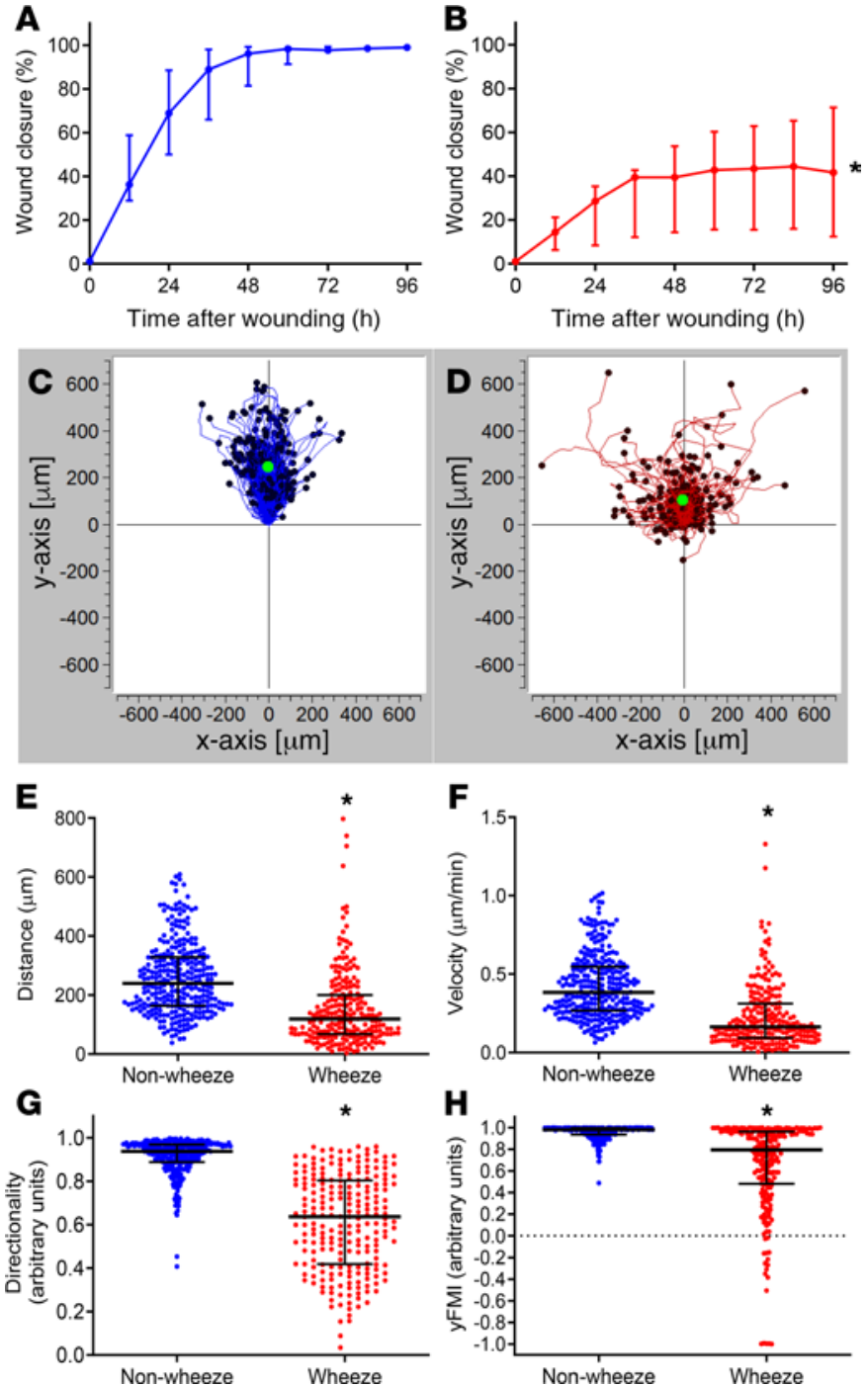

Figure 1. Defective cell migration of leading edge cells in pAEC of children with wheeze. (A) Cultures from children without wheeze had the capacity to repair by 72 hours after wounding. (B) In contrast, cultures from children with wheeze failed to close the wound by 96 hours after wounding. (C) Leading edge PAEC of children without wheeze responded to the scratch wounding stimulus by migrating directionally, toward the center of the wound site. (D) Leading edge pAEC of children with wheeze showed a dysregulated response to wounding, where some cells migrated into the wound site in an uncoordinated manner and other cells did not migrate very far into the wound and even migrated backward into the leading edge. The green dot represents the mean center of mass of the endpoints of all tracked cells. (E and $\mathbf{F}$ ) Leading edge PAEC from children without wheeze migrated far (E) and fast (F) into the wound site by 10 hours after wounding, although response to wounding was varied. However, leading edge cells of children with wheeze migrated shorter average distances $(\mathbf{E})$ and at slower velocity $(\mathbf{F})$ than their nonwheezing counterparts $(P<0.050)$. ( $(\mathbf{G}$ and $\mathbf{H})$ Notably, leading edge cells of children without wheeze migrated directionally (G) and collectively into the center of the wound, as shown with high $y$ axis forward migration index (yFMI) values (H). Conversely, leading edge PAEC of children with wheeze demonstrated migration trajectories with significantly less directionality $(\mathbf{G})$ and yFMI $(\mathbf{H})$, indicating a loss of coordination in their response to wounding. Cell migration trajectory data were generated from 296 and 228 leading edge cell tracks of children with wheeze $(n=14)$ and without wheeze $(n=9)$, respectively. All experiments were completed in 2 technical replicates. The data were represented as median $\pm I Q R$, ${ }^{*} P<0.050$, Mann-Whitney $U$ test.

to 24 canonical pathways (Supplemental Table 5), where PI3K/Akt, cholecystikynin/gastrin, TLR, acute phase response, and IL-6 signaling were the top 5 downregulated pathways (Figure 3C). When the PI3K/Akt signaling pathway was interrogated further, a number of genes (17 upregulated and 17 downregulated) were found to be significantly DE and highly interconnected, suggesting this pathway's key biological role during wound repair (Figure 3D). 

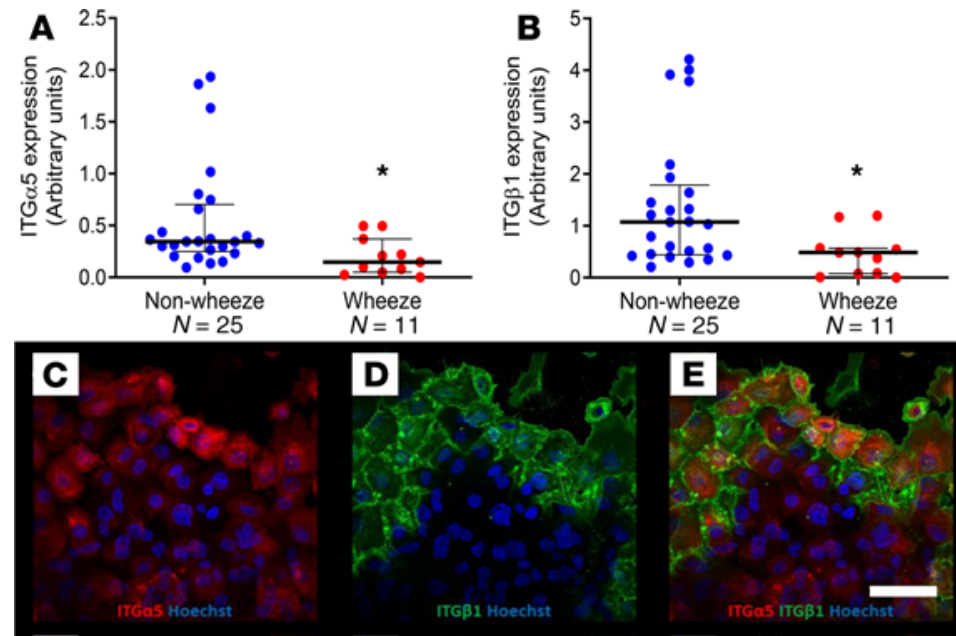

$N=11$
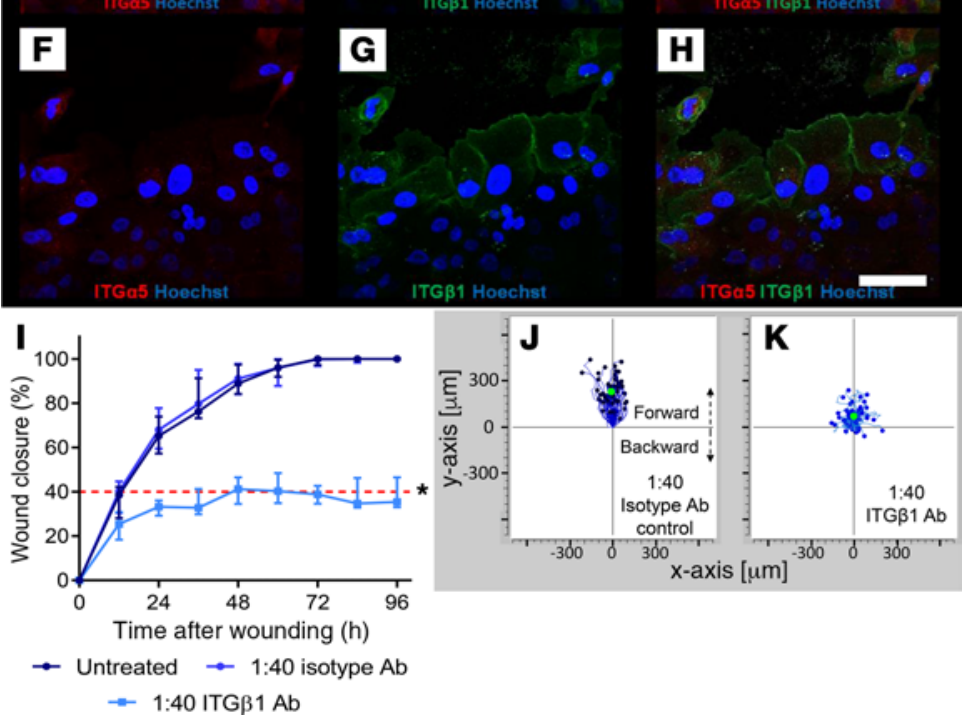

Figure 2. Role of integrins in pAEC from children with and without wheeze. ( $A$ and $B$ ) Protein expression of integrin subunits $\alpha 5$ (A) and $\beta 1$ (B) were found to be significantly lower in PAEC from children with wheeze compared with their nonwheezing counterparts. (C-E) Strong immunofluorescence staining of both integrin $\alpha 5$ (C) and $\beta 1$ (D) on leading edge PAEC from children without wheeze was demonstrated that was almost undetectable in sites distal to the wound (E). (F-H) In contrast, pAEC cultures of children with wheeze exhibited weak integrin $\alpha 5$ (F) and $\beta 1$ (C) staining along the leading edge cells $\mathbf{( H )}$. The slides were counterstained with Hoechst nuclear stain (blue). Representative images of samples from 5 children with wheeze and 5 children without wheeze (C-H). Oil immersion objective, optical magnification of 600x, imaged using 60x objective and 10x ocular lens; NA 1.4. Scale bar: $50 \mu \mathrm{m}$. (I) Blocking $\beta 1$ integrin ( $1: 40$ dilution, IgG1, P5D2) function significantly reduced $\mathrm{PAEC}$ wound closure rates to similar rates observed in their

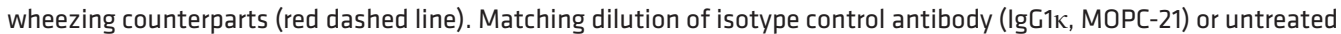
PAEC from children without wheeze reached full closure by 72 hours after wounding. $n=4$ children. Median $\pm I Q R,{ }^{*} P<$ 0.050 , Mann-Whitney $U$ test. (J) Cultures treated with isotype antibody (1:40 dilution) displayed comparable migration patterns with untreated pAEC from children without wheeze. (K) However, cultures treated with 1:40 dilution of anti- $\beta 1$ integrin antibody in culture media migrated less far into the wound, lacking cell directionality and specificity toward the wound center. Individual cell tracks were transposed so that each track had its start at the origin. $n=60$ tracks from 4 children without wheeze (I-K).

We investigated global transcriptional expression patterns by leading edge cells from primary cultures of children with and without wheeze at 24 hours after wounding by employing prior knowledge built into the upstream regulator analysis package within ingenuity systems (Ingenuity Pathway Analysis). Upstream regulator analysis identified 2 molecules to be activated and 33 to be inhibited that have previously been shown to alter gene expression of either integrin subunit individually or both subunits concurrently (Supplemental Table 6). Further analysis revealed upstream regulators of integrin $\alpha 5$ and/or $\beta 1$ expression, including several growth factors and signaling molecules, such as PDGF BB, HRAS, PI3K (complex), MAP2K1, ERBB2, and Akt (Figure 3E and Supplemental Table 6). We also conducted a comprehensive 
A

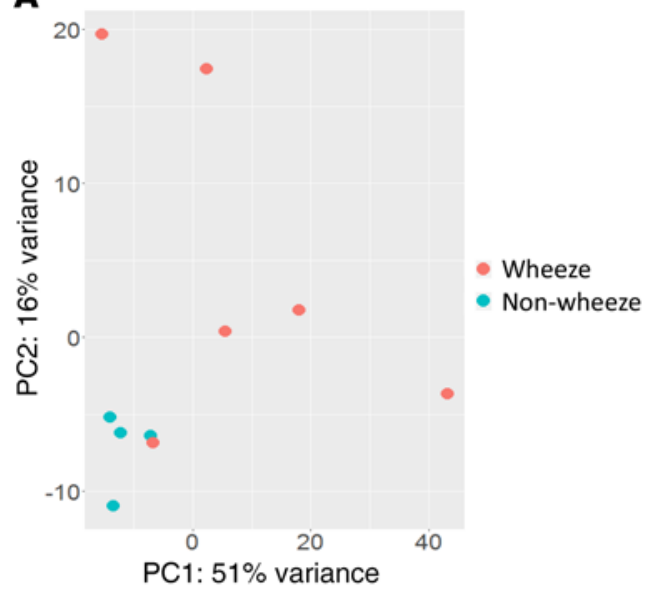

C

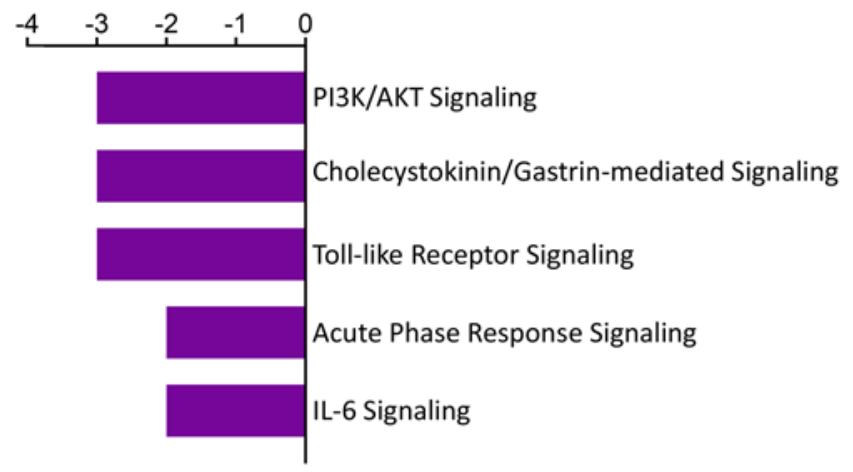

B

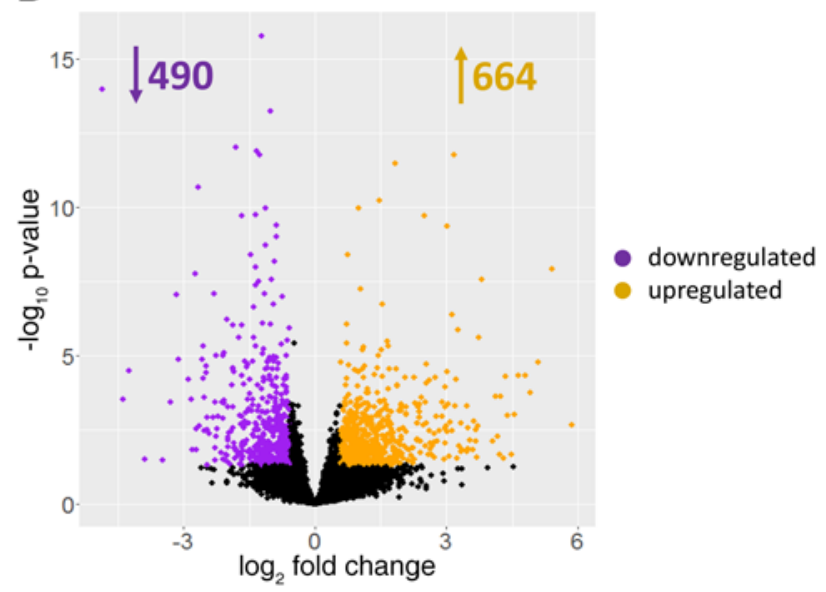

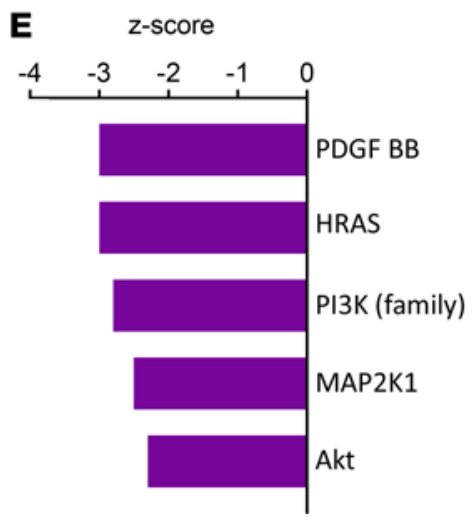

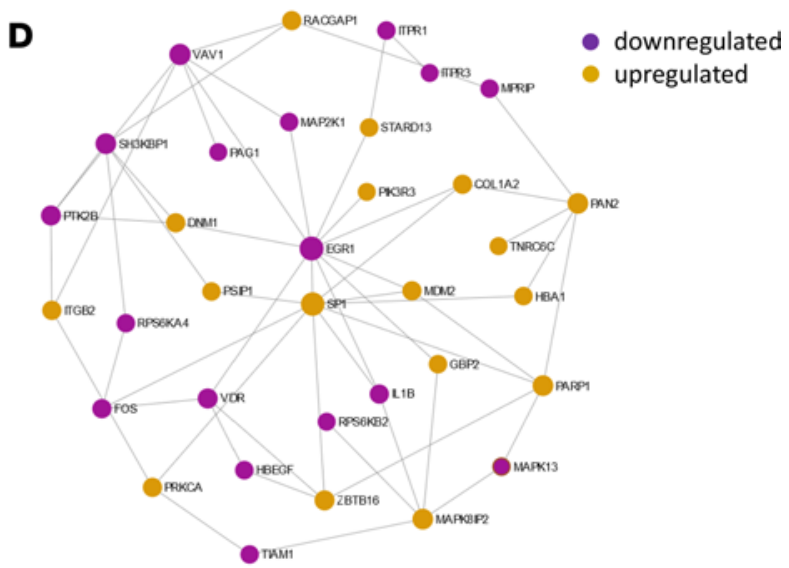

$\mathbf{F}$

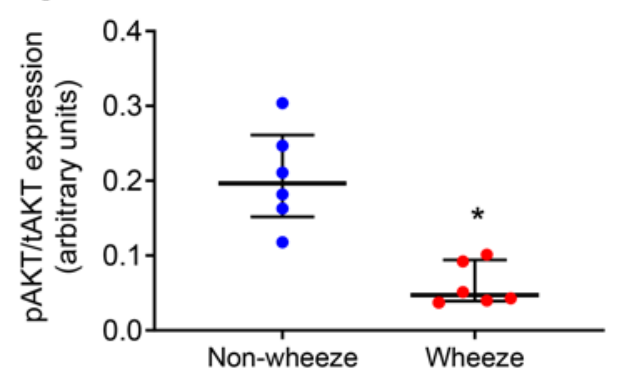

Figure 3. Transcriptional response to in vitro pAEC wounding. (A) Two-dimensional principal component analysis (PCA) plot displays samples from children without wheeze (blue; $n=4$ children) and samples from children with wheeze (red; $n=6$ children) to cluster separately, indicating large transcriptional differences. (B) Volcano plot. Scattered points represent genes: the $x$ axis is the log -transformed fold-change values per gene for leading edge samples at 24 hours after wounding from children with wheeze relative to matching nonasthmatic controls. Log $_{10}$-transformed $P$ values above 1.3 were considered statistically significant (Benjamini-Hochberg-adjusted $P<0.050$ ). Orange dots are genes significantly upregulated (log foldchange $>0.58$, adjusted $P<0.050$ ), and purple dots are significantly downregulated genes (log fold-change $<-0.58)$ in samples from children with

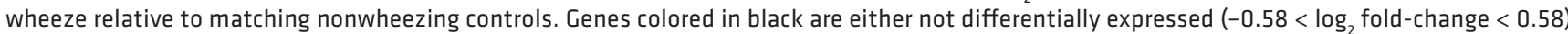
or did not reach statistical significance (adjusted $P>0.050$ ). (C) Top 5 downregulated canonical pathways associated with differentially expressed genes in leading edge pAEC at 24 hours after wounding from children with wheeze compared with corresponding samples of nonwheezing children. (D) Network map of genes associated with PI3K/Akt signaling pathway. Genes are highlighted as upregulated (orange) or downregulated (purple). (E) Top 5 upstream transcriptional regulators of integrin $\alpha 5$ and $\beta 1$ ranked with predicted $Z$ scores. All upstream regulators were determined to be statistically significant (adjusted $P<0.050)$. (F) Lower levels of activated Akt (phosphorylated Ser473) in pAEC from children with wheeze $(n=6$ children) compared with their nonasthmatic counterparts ( ${ }^{*} P<0.050$; Mann-Whitney $U$ test; $n=6$ children). Levels of pAKT were normalized to total Akt levels, and data were represented as median \pm IQR. 
review of the literature to further refine the candidate regulator from this list known to be involved in airway epithelial cell migration and repair. The PI3K pathway — and its downstream signaling effector, Akt - were identified as the most extensively studied in the literature for their roles in airway cell migration and repair regulation (Supplemental Table 7).

Inhibition of PI3K/Akt signaling abrogates $p A E C$ migration and repair. Given our transcriptomic analysis and corroboration by prior knowledge (Supplemental Table 7), we explored whether dysregulated signaling via the PI3K/Akt pathway occurs in pAEC from children with wheeze, thereby reducing the expression of integrin subunits $\alpha 5$ and $\beta 1$ and impairing the capacity of cells to migrate and repair efficiently. Protein analysis of the Akt pathway determined that, although total Akt expression was abundantly expressed, phosphorylated Akt at serine 473 was significantly reduced in pAEC from children with wheeze compared with nonwheezing controls $(P<0.050$, Figure $3 \mathrm{~F})$. To determine the effect of reduced PI3K/Akt signaling on wound repair, we initially treated pAEC cultures from nonwheezing children with wortmannin, a broad-spectrum PI3K inhibitor $(33,34)$. As expected, the levels of activated Akt were significantly reduced following treatment with wortmannin $(P<0.050$, Supplemental Figure $4 \mathrm{~A})$. This resulted in significant reduction in expression of both $\alpha 5(P<0.050$, Supplemental Figure $4 \mathrm{~B})$ and $\beta 1(P<0.050$, Supplemental Figure $4 \mathrm{C})$ integrin subunits. Finally, inhibition of PI3K signaling resulted in concentration-dependent attenuation of closure rates $(P<0.050$, Supplemental Figure 4D) and migration of leading edge cells $(P<$ 0.050; Supplemental Figure 4, E-M).

We replicated key experiments using a specific inhibitor of Akt, MK2206 (Figure 4). Treatment of pAEC from nonwheezing children with increasing concentrations of MK2206 $(0.01-1 \mu \mathrm{M})$ also resulted in a concentration-dependent inhibition of Akt activation $(P<0.050$, Figure $4 \mathrm{~A})$ and reduced expression of integrin $\alpha 5$ on the cell membrane in as little as 12 hours $(P<0.050$, Figure $4 \mathrm{~B})$. Conversely, Akt inhibition did not significantly affect integrin $\beta 1$ expression following 12 - or 48 -hour treatment ( $>0.050$, Figure $4 \mathrm{C}$ ). Treatment with MK2206 also reduced wound closure $(P<0.050$, Figure 4D) and leading edge cell migration, reflected by distance migrated, velocity, directionality, and forward migration index (FMI) compared with vehicle-treated cultures from children without wheeze (all $P<0.050$; Figure 4, E-M), recapitulating repair responses of cells from wheezers.

SC79 targets the Akt/integrin a 5B1 pathway to enhance pAEC migration and repair in childhood wheeze. To determine whether activation of Akt could enhance integrin $\alpha 5 \beta 1$ expression, migration, and wound repair, pAEC cultures of wheezing children were treated with increasing concentrations $(0.5-20 \mu \mathrm{M})$ of the Akt activator, SC79 (Figure 5). SC79 treatment augmented Akt signaling (2-20 $\mu \mathrm{M}, P<0.050$, Figure 5A) and increased protein expression of both integrin subunits $\alpha 5(0.5-20 \mu \mathrm{M}, P<0.050$, Figure $5 \mathrm{~B})$ and $\beta 1(0.5-5$ $\mu \mathrm{M}, P<0.050$, Figure 5C). Importantly, SC79 treatment significantly enhanced wound closure of pAEC of children with wheeze in a concentration-dependent manner compared with vehicle and untreated controls $(P<0.050$, Figure 5D). Improved migration was reflected in greater distance migrated, increased velocity, and enhanced directionality, toward the center of the wound $(P<0.050$, Figure $5, \mathrm{E}-\mathrm{M})$.

Celecoxib targets the PI3K/Akt-integrin $\alpha 5 \beta 1$ axis to enhance repair of epithelial cells from children with wheeze. Celecoxib, an FDA-approved selective COX2 inhibitor used in the treatment of rheumatoid arthritis, juvenile arthritis, and pain management, has been reported to activate PI3K/Akt $(35,36)$. Therefore, we tested whether celecoxib would enhance airway epithelial cell repair in wheezers through the activation of PI3K/Akt signaling and integrin $\alpha 5$ and $\beta 1$ expression. Furthermore, we also tested the dimethyl-celecoxib analogue, which lacks inhibitory activity against COX2, to demonstrate that effects on epithelial repair were independent of COX2 inhibition.

As expected, celecoxib and dimethyl-celecoxib treatment activated PI3K/Akt signaling $(P<0.050$, Figure 6A, Supplemental Figure 5A, Supplemental Figure 6A) and upregulated expression of $\alpha 5(P<$ 0.050, Figure 6B, Supplemental Figure 5B, Supplemental Figure 6B) and $\beta 1(P<0.050$, Figure 6C, Supplemental Figure 5C, Supplemental Figure 6C) integrin subunits in pAEC from children with wheeze. Importantly, exposure to either celecoxib or dimethyl-celecoxib enhanced epithelial repair in a concentration-dependent manner (all concentrations $P<0.050$, Figure 6D, Supplemental Figure 5D, Supplemental Figure 6D). Both compounds stimulated repair by enhancing leading edge cell migration (all concentrations $P<0.050$; Figure 6, E-G; Supplemental Figure 5, E-G; Supplemental Figure 6, E-G). Cultures of pAEC treated with either drug migrated longer distances $(P<0.050$, Figure $6 \mathrm{H}$, Supplemental Figure 5J, Supplemental Figure 6J), at higher velocity $(P<0.050$, Figure 6I, Supplemental Figure 5K, Supplemental Figure $6 \mathrm{~K})$ and directionality $(P<0.050$, Figure 6J, Supplemental Figure 5L, Supplemental Figure 6L), 

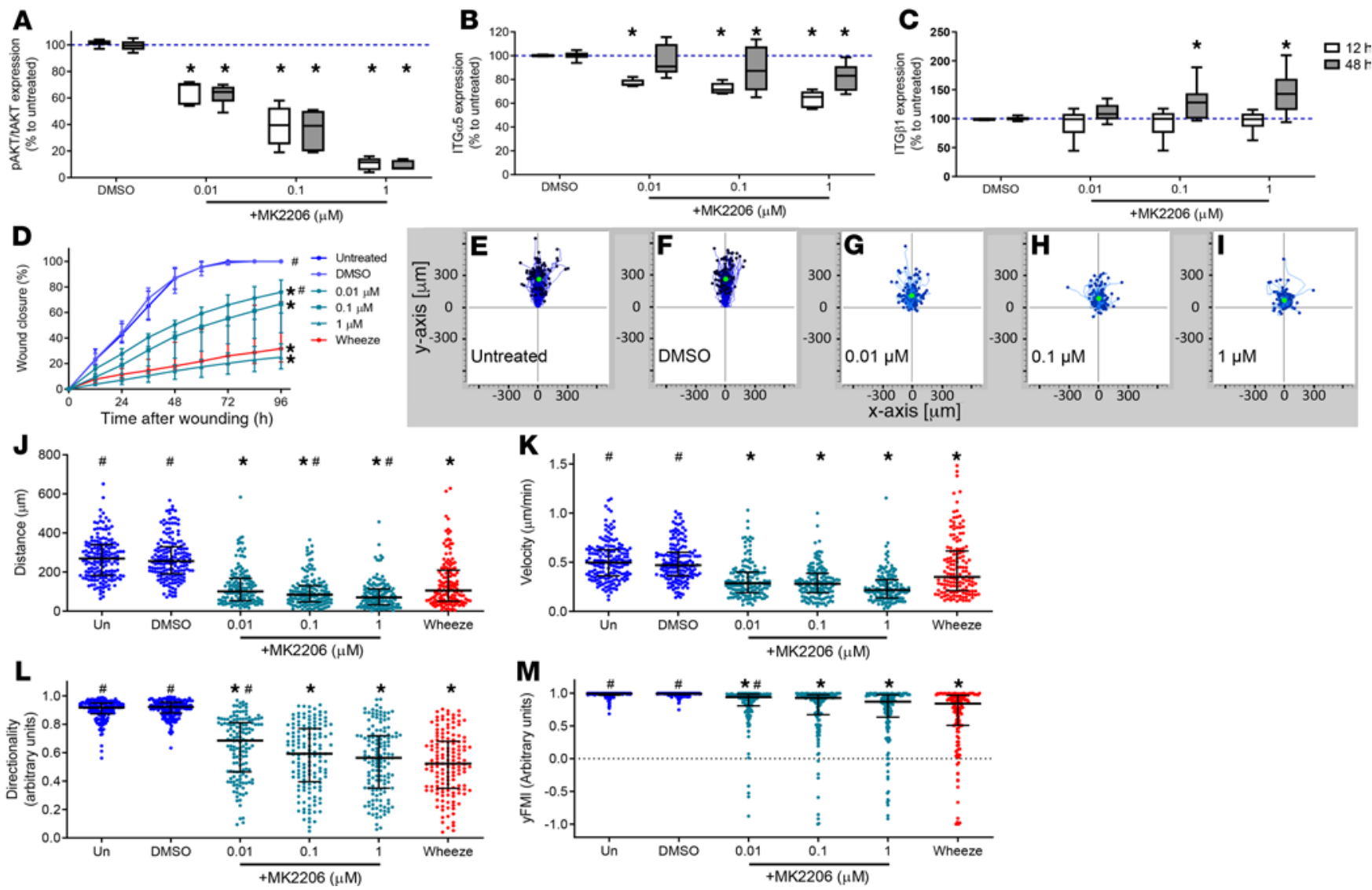

Figure 4. Inhibition of Akt signaling abrogates pAEC repair and integrin expression. pAEC from children without wheeze were treated with different concentrations (0.01, 0.1, $1 \mu \mathrm{M}$ ) of the specific Akt inhibitor MK2206. (A) MK2206 inhibited phosphorylation of Akt (serine residue 473 ) at 12 and 48 hours after treatment. (B and C) Inhibition of Akt in pAEC from children without wheeze resulted in significant reduction of integrin subunit $\alpha 5$ (B) cell membrane expression in a concentration-dependent manner at 12 and 48 hours after MK2206 treatment. Although no differences were observed in cell membrane integrin subunit $\beta 1$ at 12 hours following Akt inhibition an increase was seen at 48 hours following treatment compared to vehicle control (C). (D) Treatment of pAEC cultures from nonwheezing children with MK2206 at the time of scratch wounding resulted in a concentration-dependent reduction in closure rates, although DMSO vehicle $(0.05 \% \mathrm{v} / \mathrm{v})$ control was not significantly altered compared with untreated cultures. (E-M) Although treatment of pAEC from nonwheezing children with $0.05 \%(\mathrm{v} / \mathrm{v})$ DMSO vehicle control had no effect on cell migration (E and F), MK2206 treatment attenuated cell migration in a concentration-dependent manner (G-I) by inhibiting distance migrated (J), velocity (K), directionality (L), and centrality (yFMI, $\mathbf{M})$. All experiments were completed with pAEC cultures from 6 children without wheeze, and data were represented as either box and whisker (min/max) or dot plots with median \pm IQR. Statistical differences between treatment and untreated control $\left({ }^{*} P<0.050\right)$ or wheezing group $\left({ }^{*} P<0.050\right)$ were determined using 2-way Kruskal-Wallis ANOVA with Dunn's post hoc test for multiple comparisons.

toward the wound center $(P<0.050$, Figure 6K, Supplemental Figure 5M, Supplemental Figure 6M) compared with vehicle-treated or untreated pAEC from children with wheeze $(P<0.050$; Figure 6, E-K; Supplemental Figure 5, E-M; Supplemental Figure 6, E-M).

Corroboration of a defective $\mathrm{PAEC}$ repair signature with independent chronic airway disease cohorts. In the absence of a suitable preclinical animal model of asthma or of intrinsic epithelial dysregulation of the PI3K-integrin $\alpha 5 \beta 1$ axis (37), we conducted meta-analysis of relevant published transcriptomic data sets. We interrogated data sets for evidence of these pathways being implicating in early-life wheeze or asthma, as well as identified who would potentially benefit from celecoxib treatment. Specifically, we targeted our comparisons to data sets using ex vivo nasal samples from adults with physician-diagnosed stable, mild asthma (38) or from viral-induced acute wheeze exacerbations in children (39). Comparison of our defective lower airway pAEC repair signature to the adult stable, mild asthma data set (38) identified more than 80 common DE genes (Supplemental Table 8), including genes associated with integrin and PI3K/Akt pathways like FN1, GRB2, $S R$, and $S H C 1$ (Supplemental Figure 8). Furthermore, there was a more-than $80 \%$ overlap of overrepresented pathways between the 2 data sets, such as integrin cell surface interactions and growth factor signaling (Supplemental Table 9) (38). Significantly, evaluation of a published microarray data set from a longitudinal viral-induced acute wheeze pediatric cohort (64 children) (39) identified 5 unique modules of coexpressed 

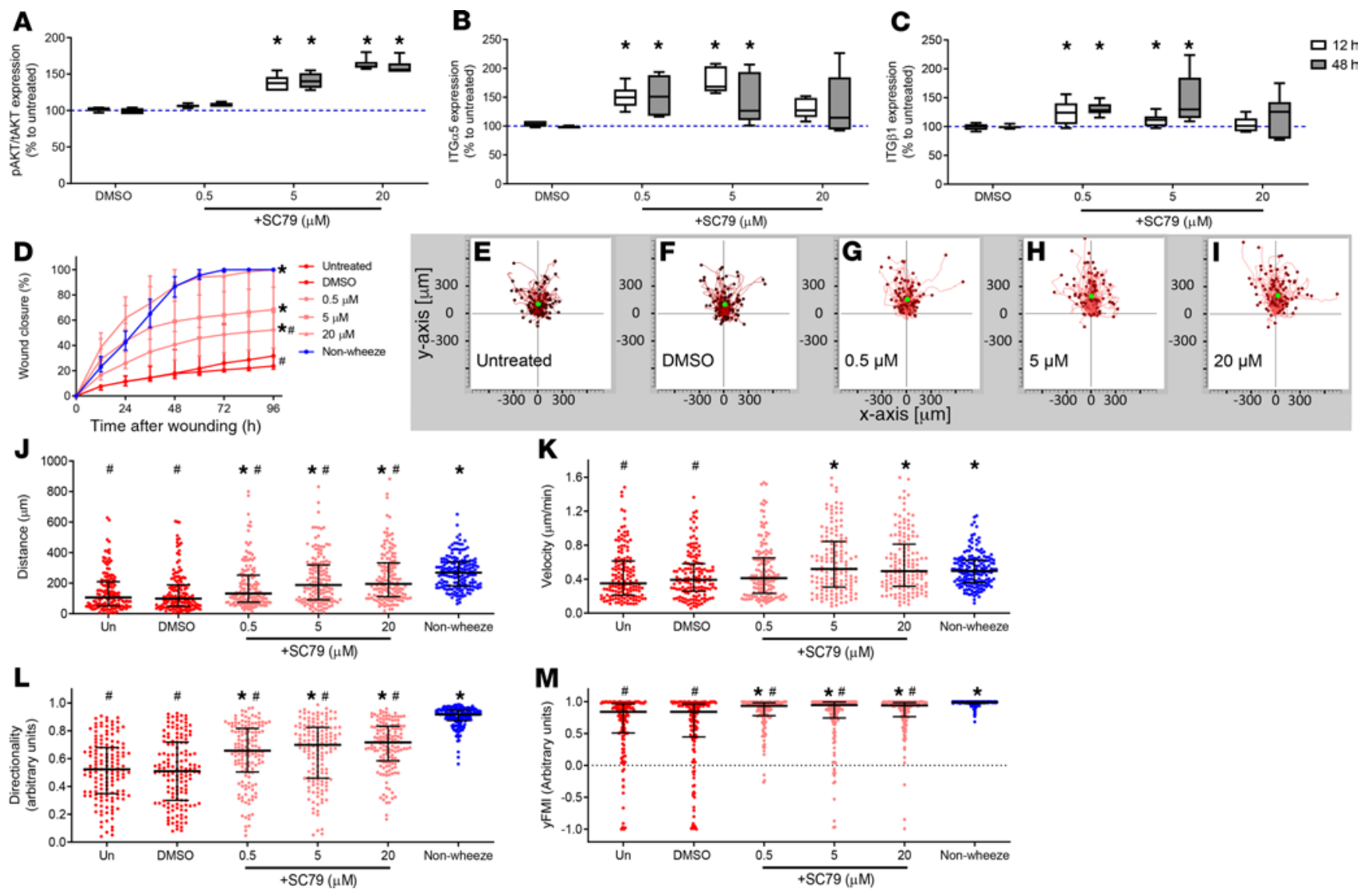

Figure 5. Activation of Akt signaling enhances repair of PAEC from children with wheeze and increased integrin $\alpha 5 \beta 1$ expression. pAEC from children with wheeze were treated with different concentrations $(0.5,5,20 \mu \mathrm{M})$ of the specific Akt activator SC79. (A) SC79 treatment resulted in phosphorylation of Akt (serine residue 473), at 12 and 48 hours after treatment. (B) Significant increase of integrin subunit $\alpha 5$ cell membrane expression was observed in PAEC from children with wheeze for all concentrations at 12 hours and $0.5,5$, and $20 \mu \mathrm{M} \mathrm{SC79}$ at 48 hours. (C) Also, integrin subunit $\beta 1$ cell membrane expression was increased in pAEC from children with wheeze treated with $0.5,2$, and $5 \mu \mathrm{M} \mathrm{SC79}$ at 12 and 48 hours. (D) Treatment of pAEC from wheezers with SC79 at the time of scratch wounding resulted in a concentration-dependent increase in closure rates, although DMSO vehicle (0.08\% v/v) control was not significantly altered compared with untreated cultures. (E-M) Although treatment of pAEC from wheezers with $0.08 \%$ ( $/ \mathrm{v}$ ) DMSO vehicle control had no effect on cell migration (E and $\mathbf{F}$ ), SC79 treatment enhanced cell migration in a concentration-dependent manner (G-I) by stimulating distance migrated (J), velocity (K), directionality (L), and centrality (yFMI, M). All experiments were completed with pAEC cultures from 6 children with wheeze, and data were represented as either box and whisker $(\mathrm{min} / \mathrm{max})$ or dot plots with median \pm IQR. Statistical differences between treatment and untreated control $\left({ }^{*} P<0.050\right)$ or nonwheezing group $\left({ }^{*} P<0.050\right)$ were determined using 2 -way Kruskal-Wallis ANOVA with Dunn's post hoc test for multiple comparisons. The wound closure (D) and cell migration parameters (J-M) for the untreated wheeze and nonwheeze groups were also presented in Figure 4 and were utilized for baseline response purposes.

genes (Figure 7A). The association of the identified modules to defective pAEC wound repair and recurrence of wheeze exacerbations was assessed in order to identify if therapeutic targeting in this pediatric cohort would be effective at reducing the number of exacerbations. Indeed, one module of genes was found to associate significantly with recurrence of wheeze exacerbations and defective wound repair (Figure 7, B and C; Supplemental Table 10). In fact, pathway analysis identified over $45 \%$ contingency between our defective repair data set and the recurrent wheeze-associated gene module, with overrepresented pathways also including integrin cell surface interactions and growth factor signaling (Supplemental Table 11). Network analysis of the identified gene module identified highly interconnected hubs like GRB2, PIK3AP1, ITGA5, and TLN1, which are known to be involved in PI3K/Akt signaling and integrin pathways (Figure 7D). Our hypothesis that defective pAEC repair through dysregulated PI3K/Akt signaling is implicated in early-life wheeze and asthma is identified via meta-analysis of relevant publicly available human gene expression data. Furthermore, these observations indicate that defective PAEC wound repair may persist in adult asthma. Thus, targeting of the PI3K-integrin $\alpha 5 \beta 1$ axis, particularly in early life, could reduce wheeze frequency, impact on disease trajectory, and persistence of early-onset asthma into adulthood. 

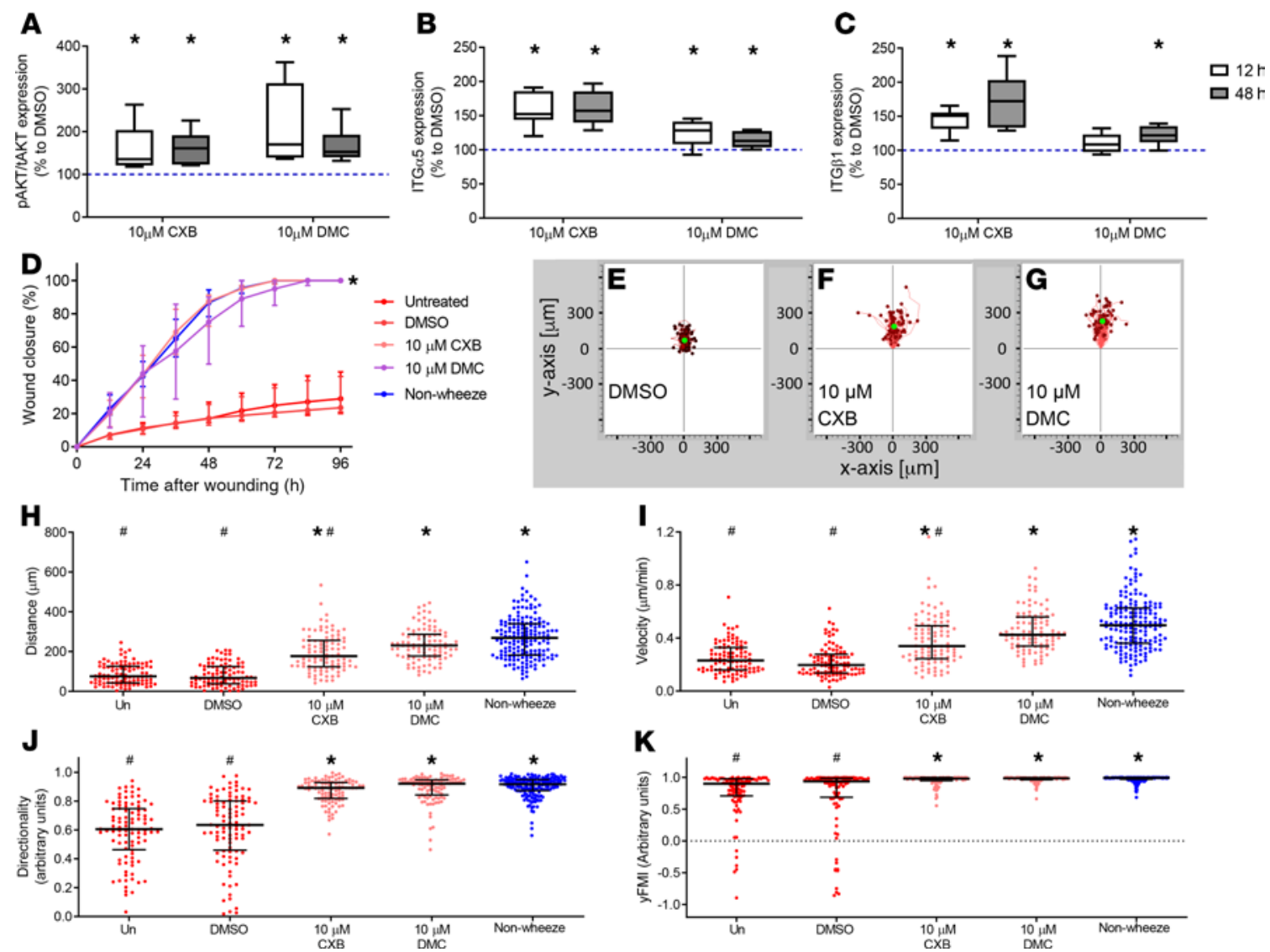

Figure 6. Repair of pAEC from children with wheeze is restored by celecoxib and dimethyl-celecoxib treatment via the PI3K/Akt-integrin $\alpha 5 \beta 1$ axis. PAEC from children with wheeze were treated with $10 \mu \mathrm{M}$ of COX2 inhibitor celecoxib or its analogue, dimethyl-celecoxib. (A) Celecoxib and its analogue treatment resulted in phosphorylation of Akt (serine residue 473) at 12 and 48 hours after treatment. (B and C) Significant increase of integrin subunits $\alpha 5$ (B) and $\beta 1$ (C) cell membrane expression was observed in treated cultures from children with wheeze at both 12 and 48 hours. (D) Treatment of pAEC cultures from wheezers with celecoxib or dimethyl-celecoxib at the time of scratch wounding resulted in complete repair comparable with their nonwheezing counterparts. (E-K) Although treatment of pAEC from wheezers with $0.13 \%(\mathrm{v} / \mathrm{V})$ DMSO vehicle control had no effect on cell migration (E), celecoxib or dimethyl-celecoxib treatments enhanced cell migration (F and $\mathbf{G})$ by stimulating distance migrated (H), velocity (I), directionality (J), and centrality (yFMI, K). All experiments were completed with pAEC cultures from 6 children with wheeze, and data were represented as either box and whisker (min/max) or dot plots with median \pm IQR. Statistical differences between treatment and untreated control $\left({ }^{*} P<0.050\right)$ or nonwheezing group $(\# P<0.050)$ were determined using 2-way Kruskal-Wallis ANOVA with Dunn's post hoc test for multiple comparisons. The wound closure (D) and cell migration parameters (J-M) for the untreated nonwheeze groups were also presented in Figure 4 and were utilized for baseline response purposes.

\section{Discussion}

With a focus of identifying therapeutic targets prior to persistent asthma development, we assessed the reparative capacity of the airway epithelium of young children, including pre-school-aged children with wheeze. As with our previous observations of defective airway epithelial cell repair in school-aged children with physician-diagnosed asthma $(13,15,27)$, we now report defective airway epithelial cell repair to also associate with recurrent wheeze in pre-school-aged children. We have demonstrated that aberrant cell migration underpins the defective repair process. Given our previous studies that indicate that exogenous addition of $\mathrm{FN}$ improves but does not provide complete restitution of cultures from children with physician-diagnosed asthma (27), we turned our attention to FN-binding integrins. In the current study, we show reduced expression of integrin $\alpha 5$ and $\beta 1$ in the airway epithelium of children with wheeze at baseline and following wounding compared with children without wheeze. Using whole cell transcriptomics, we identified several upstream transcriptional regulators of integrin $\alpha 5$ and $\beta 1$ expression, of which the PI3K/Akt signaling pathway was the top predicted regulator and was downregulated in children in cells from children with wheeze. Furthermore, we confirmed reduced Akt activation in pAEC from children with wheeze. Significantly, inhibition of this pathway in pAEC from nonwheezing controls resulted in decreased expression of integrin $\alpha 5 \beta 1$, reduced migration, and defective repair, mimicking what was observed in pAEC from children with a wheeze phenotype. Conversely, activation of the PI3K/Akt pathway in pAEC from 

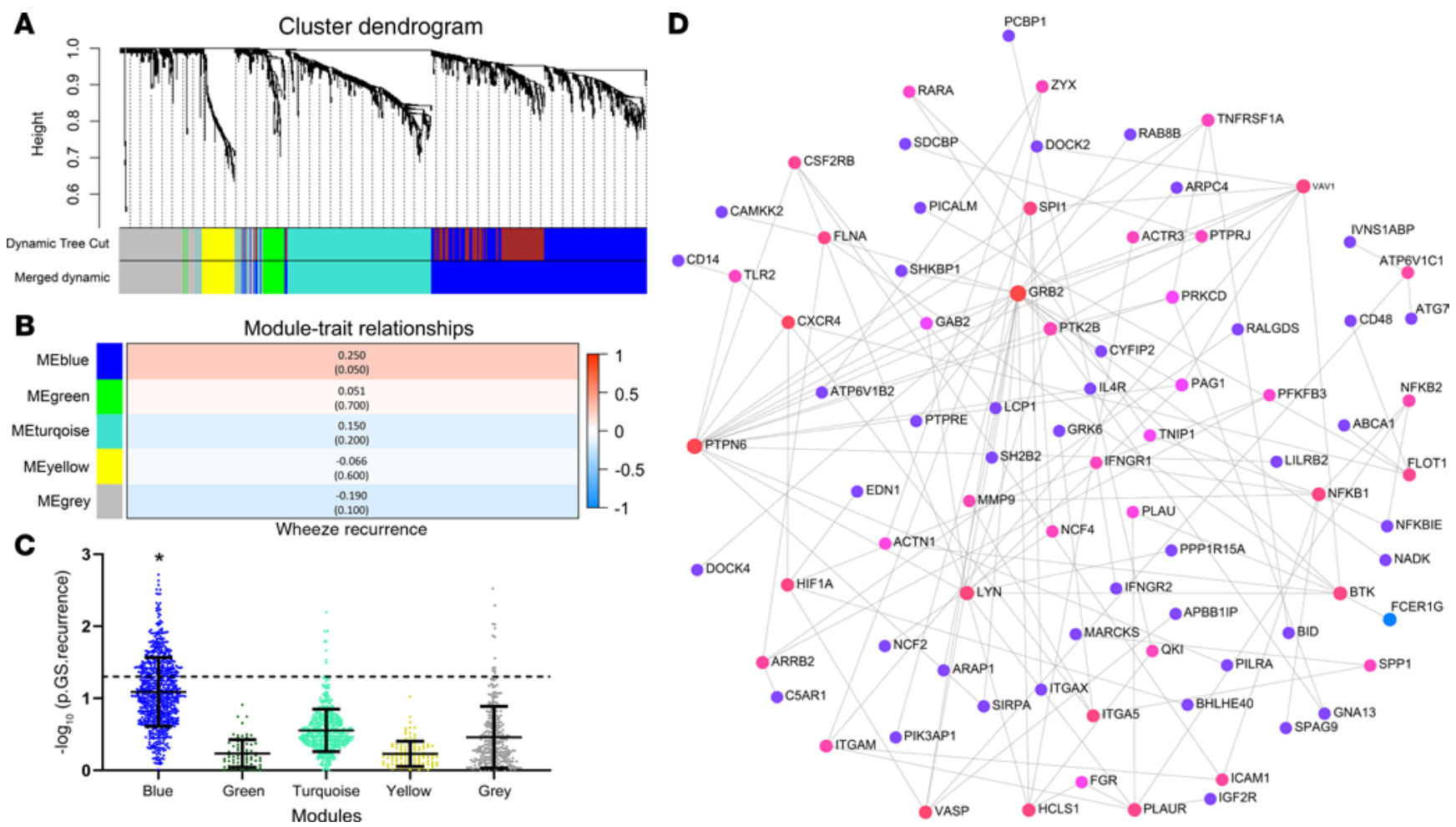

Figure 7. Evaluation of the defective PAEC repair gene signature in published transcriptomic data sets. (A) Weighted gene coexpression network analysis (WGCNA) cluster dendrogram. Hierarchical cluster analysis was conducted to detect gene coexpression clusters with corresponding color assignments using data from 64 children with or without recurrent wheeze. Each color represents a module in the constructed gene coexpression network by WCCNA. (B) WGCNA modules and sample trait heatmap. Using the default parameter settings and genes filtered based on probeset concordance $(n=1737)$, 5 gene modules were identified to correlate with recurrence of respiratory wheeze. Data are presented with the correlation coefficient ( $P$ value), where positive correlations are red, and negative correlations are blue. (C) Weighted gene coexpression network analysis calculation of gene significance (GS) to sample trait of interest, recurrence of wheeze, in each gene module. The blue module had an overrepresented number of genes associating with wheeze recurrence. (D) Minimum network map of blue module genes that strongly associate with wheeze recurrence in the pediatric acute wheeze data set. Genes are highlighted as highly interconnected (pink) or weakly interconnected (purple) according to known protein/protein interactions from published studies (prior knowledge). Size of nodes indicate a larger number of connections.

children with wheeze increased integrin $\alpha 5 \beta 1$ expression and enhanced epithelial cell migration and repair following wounding, recapitulating a normal response. We demonstrated that FDA-approved celecoxib and its experimental analogue, dimethyl-celecoxib, enhanced integrin expression and restored leading edge cell migration, resulting in complete epithelial repair in cultures from children with wheeze.

In the absence of a suitable animal model of asthma or of epithelial dysfunction due to PI3K/Aktintegrin $\alpha 5 \beta 1$ downregulation (37), we interrogated published ex vivo pAEC transcriptomic data sets for evidence of these pathways being implicated in early-life wheeze or asthma. We discovered that defective wound repair and dysregulated PI3K/Akt expression were associated with: (a) adult asthma (38), (b) acute wheeze exacerbations, and (c) wheeze recurrence in children presenting to hospital emergency department (39). Our observations of defective epithelial repair contributing to the development of respiratory symptoms are also supported by McErlean and colleagues (16), who identified inadequate nasal epithelial repair to differentiate adult asthmatics that experienced acute respiratory exacerbation. The presence of signatures of aberrant epithelial repair in both young children with recurrent wheeze and adults with more persistent symptoms implies a trajectory that could be mitigated by early intervention to restore normal epithelial function. The in vitro normalization of epithelial cell migration and repair by celecoxib and its analogue without COX-2 inhibitory activity suggests an easily testable hypothesis and indicate that a safe, early intervention is feasible.

Celecoxib is already approved for use in children with juvenile arthritis and could be repurposed for treatment of children with wheeze relatively expediently, requiring only Phase II/III safety and efficacy clinical trials. Although celecoxib has been previously associated with an increased risk of myocardial infarction in adults, these adverse cardiovascular events appear less frequently than with other classical NSAIDs like Ibuprofen (40) 
and have not been reported in children. Furthermore, adult asthma patients with aspirin-exacerbated respiratory disease can safely be treated with celecoxib (41). Importantly, no acute lung function decline has been observed following selective COX2 inhibition with celecoxib in asthmatics $(42,43)$. Thus, this may highlight celecoxib as a new area for therapy and for further investigation in adults with aspirin-exacerbated respiratory disease to improve respiratory outcomes. Our study highlights that the capacity of celecoxib to aid repair in pAEC from children with wheeze is independent of COX2 inhibition. Unlike our findings, previous work in the oncology literature has suggested that celecoxib treatment of cancer cell lines may inhibit PI3K/Akt signaling at high micromolar concentrations $(44,45)$. This discrepancy may highlight dose-dependent and cell-specific responses to celecoxib treatment in vitro. Our observations warrant future investigations on the modulation of the PI3K/ Akt/integrin $\alpha 5 \beta 1$ axis in the airway epithelium to aid barrier restitution and limit inflammation following injury. In addition, further studies are needed for the therapeutic evaluation of celecoxib in the clinical setting and for consideration of the development of aerosolized formulations $(46,47)$, or analogues, to improve bioavailability in vivo and enhance epithelial repair in the airways of patients with wheeze or asthma.

The extensive patient cohort used in this study reflects the general pediatric population; therefore, most children with wheeze providing cells had mild but persistent symptoms. Despite the relatively simple phenotyping of the cohort, we have observed abnormal epithelial cell repair in cultures from children with recurrent wheeze and/or physician-diagnosed asthma used in this and previous studies $(15,27)$. This raises the possibility that a heterogenous group of wheezing and respiratory disorders in young children might represent the clinical expression of an underlying vulnerable epithelial endotype. The reported observations are unlikely to be as a result of therapy, since none were receiving inhaled corticosteroids or bronchodilators for at least 1 month prior to recruitment into the study. In addition, we utilized standard culture and conditional reprogramming methods that conserve the wound repair phenotypes of pAEC (48). Proteins of interest that were $\mathrm{DE}$ in cells traditionally cultured in monolayers were similarly expressed in the conditionally reprogrammedderived cells. These findings further support our hypothesis that intrinsic abnormalities exist in the airway epithelium of children that associate with established clinical phenotypes, such as recurrent wheeze and asthma.

In summary, we have identified a potentially novel vulnerable epithelium endotype implicated in young children with wheeze that is characterized by abnormal capacity of airway epithelial cells to migrate during wound reepithelization. Specifically, dampened signaling via the PI3K/Akt pathway leads to reduced protein expression levels of $\alpha 5$ and $\beta 1$ integrin subunits, and stimulation of the PI3K/Akt pathway restores $\alpha 5$ and $\beta 1$ integrin protein expression and promotes normal migration and wound repair. Enhancing repair of damaged airways in individuals with wheeze and asthmatics could minimize the impact of virus infections, reduce chronic inflammation, and minimize airway damage and wheeze recurrence. This study has provided further insight regarding important intrinsic epithelial vulnerability in childhood wheeze and asthma, identified FDA-approved celecoxib as a potential therapy, and suggested a rationale for investigating epithelium-centered therapies in young children with wheeze. These observations warrant a clinical trial to assess efficacy of celecoxib to accelerate airway epithelial repair and reduce symptoms of wheeze in children.

\section{Methods}

\section{Study participants and sample collection}

Two cohorts were used in this study: 91 (59 male) children with recurrent wheeze and 110 (67 male) children with no history of wheeze (Supplemental Table 12). Subjects were between 1.2 and 17.7 years of age, were undergoing elective surgery for non-respiratory-related conditions, and had no clinical symptoms of bacterial or viral chest infection within 2 weeks preceding recruitment. Children with an existing bacterial or viral chest infection were excluded. The wheeze cohort was defined according to expression of respiratory wheeze and/or physician diagnosis of asthma in school-aged children. Subjects experienced recurrent and persistent respiratory wheeze in the last 3 months, as well as over 12 months prior to recruitment into the research program. Asthma was defined as physician diagnosed, based upon physician-documented wheezing episodes in the 12 months preceding their recruitment and confirmed by positive responses on the International Study of Asthma and Allergies in Children (ISAAC) and American Thoracic Society (ATS) respiratory questionnaires $(49,50)$. Although stable at the time of sampling, all children with recurrent wheeze had mild respiratory symptoms, such that none had received glucocorticosteroids (inhaled or oral) or $\beta$-agonists at least 1 month prior to recruitment and airway sampling. Tracheobronchial AEC were obtained via translaryngeal, nonbronchoscopic brushings of children through an endotracheal tube, as previously described $(11,27,51,52)$. 


\section{Reagents}

BSA, FBS, bovine hypothalamus acetone power, hydrocortisone, recombinant human epidermal growth factor, epinephrine hydrochloride, FN, rat tail type I collagen, triiodothyronine, transferrin, trans retinoic acid, trypsin, gentamicin, wortmannin solution, and SC79 were obtained from MilliporeSigma, and MK2206 was provided by Raelene Endersby (Telethon Kids Institute, Perth, W.A., Australia). Bronchial epithelial basal medium (BEBM) and bronchial epithelial cell growth medium (BEGM) were purchased from Lonza. Penicillin G, RPMI-1640 media, streptomycin sulfate, amphotericin B, and L-glutamine were purchased from Invitrogen. Ultroser G was supplied from Ciphergen. Collagen type I and FN were purchased from BD Biosciences, and all tissue culture plasticware was purchased from Corning. Monoclonal antibodies against human integrin subunits (integrin $\alpha 5$, EPR7854 clone, ab150361; integrin $\beta 1$, P5D2

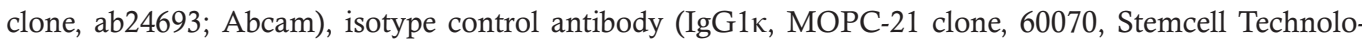
gies), phosphorylated Akt (serine residue 473, D9E clone, 4060; Cell Signaling Technology), and pan-Akt (40D4 clone, 2920; Cell Signaling Technology).

\section{Cell culture}

This study used conditional reprogramming $(48,53)$ and traditional culturing $(11,52)$ methods to establish ex vivo human pAECs from children. The remainder of ex vivo cells were used for RNA extraction and gene expression profiling as previously described (11). All cell culture experiments were performed in primary submerged monolayers in BEGM (Lonza).

\section{Wound repair assays}

Ninety-six-well Essen WoundMaker. To assess wound repair kinetics, linear scratch wounds were created using a commercial monolayer wounding kit (54). Wound closure was calculated through image analysis by the accompanying live-cell imaging system (Essen BioScience).

Leading edge cell tracking and quantification of migration parameters. Manual Tracking, a publicly available ImageJ2 plugin, was utilized for its capacity to manually track cells in 2 dimensions, the $x$ and $y$ planes (55). Time-lapse images of the first 12 hours after wounding were loaded into ImageJ2, and approximately 10-20 individual leading edge cells were arbitrarily selected from both wound margins - that is, the top and bottom margins. The selected cells were tracked over the first 12 hours after wounding, where this procedure generated $x$ and $y$ coordinates for the position of the nucleus of each tracked cell at each time point. The $x$ and $y$ coordinates of the tracked cells were exported in a tab delimited format, and all tracks were transformed to migrate up toward the $y$ axis.

Data interpretation and quantification of migration parameters. Graphical representation and analysis of the cell migration parameters was performed using the Chemotaxis and Migration Tool 2.0 (Ibidi). The $x$ and $y$ coordinates were imported into the Chemotaxis and Migration Tool 2.0, where the $x$ and $y$ coordinates of cell tracks were transformed to a common origin (coordinates 0,0 ) for downstream data analysis and visualization purposes. Data were graphically presented in various forms, including 2-dimensional trajectory plots where the origin represented the wound margin and the trajectories indicated the migration paths of individual cells over 10 hours. In addition, circular plots were used to indicate the directional frequency of tracks at each angle position, with the maxima of counts marked in red. Migration parameters of cell tracks were then quantified including (a) distance ( $\mu \mathrm{m})$, which represented the displacement of a cell from the start to its endpoint; (b) velocity $(\mu \mathrm{m} / \mathrm{min})$, which is a function of displacement over total tracking time (i.e., 600 minutes); and (c) directionality (arbitrary units), which represents a measurement of the directness or straightness of a cell trajectory and was calculated as a ratio of the cell displacement over the accumulated distance migrated. A ratio of 1 indicated absolute directional cell migration and a ratio of 0 indicated totally random migration. Since directionality is not a direct measure of chemotaxis, the determined FMI (arbitrary units) was then utilized to represent the efficiency of the leading edge cells to migrate toward the center of the wounded area. Since leading edge cells were expected to move forward toward the center of the wound, FMI along the $y$ axis (yFMI) was reported. yFMI was calculated as a ratio of the $y$ coordinate at the cell endpoint over displacement, where a high determined value indicated a high efficiency of leading edge cells to respond to the generated wound and a negative yFMI value represented a leading edge cell migrating backward, into the wound site. 
Cell viability assay

Cell viability was assessed using a 3-[4,5-dimethylthiazol-2yl]-5-[3-carboxymethoxyphenyl]-2-[4-sulfophenyl]-2H-tetrazolium inner salt (MTS) assay (Promega) (56) for wortmannin, MK2206, SC79, celecoxib, and dimethyl-celecoxib (Supplemental Figure 7).

\section{Gene expression analysis by quantitative PCR ( $\mathrm{PPCR}$ )}

The mRNA expression of FN-binding integrin subunits and housekeeping gene PPIA was determined with gene-specific primers listed in Supplemental Table 13. Gene expression was determined by using 2-step reverse transcription and real-time PCR assays, as previously described (11). Relative gene expression was calculated using the $2^{-\triangle \triangle C T}$ method by normalization to PPIA housekeeping gene and an endogenous tissue control (57).

\section{In-cell and on-cell Western assays}

The protein expression of integrin subunits $\alpha 5$ and $\beta 1$, phosphorylated Akt (serine residue 473), and total Akt was quantified via the in-cell and on-cell Western assays, as described (14). Briefly, cells were plated at $1.2 \times 10^{5} \mathrm{cells} / \mathrm{cm}^{2}$ onto 96 -well plates precoated with $\mathrm{FN}$, collagen, and BSA and maintained as described earlier $(11,48,52)$. Briefly, following relevant treatments and controls, confluent cell monolayers were fixed with $4 \%(\mathrm{w} / \mathrm{v})$ PFA for 20 minutes at room temperature and then either permeabilized with $0.1 \%(\mathrm{v} / \mathrm{v})$ Triton X-100 or incubated with PBS for 25 minutes. Cells were blocked for 90 minutes at room temperature with the Odyssey blocking buffer (PBS) (LI-COR Biosciences). Cells were then stained with rabbit anti-human integrin $\alpha 5$ (1:100; clone EPR7854, Abcam), mouse anti-human integrin $\beta 1$ (1:1000; clone P5D2, Abcam), rabbit anti-human phosphorylated Akt (serine residue 473) (1:100; clone D9E, Abcam), or mouse anti-human total Akt (pan) $\left(1: 100\right.$; clone 40D4, Abcam) for 24 hours at $4{ }^{\circ} \mathrm{C}$. Antibody binding was determined using IRDye $800 \mathrm{CW}$-conjugated goat anti-rabbit IgG (1:800; 925-32211, LI-COR Biosciences) and goat anti-mouse IgG (1:800; 925-32210, LI-COR Biosciences), as well as DRAQ5 (1:10000; DR50200, BioStatus Limited) and Sapphire700 (1:1000; 928-40022, LI-COR Biosciences) for cell number normalization, for 1 hour at room temperature with gentle shaking in the dark. Cells were then washed for an additional 5 times in PBS. Specific antibody staining for protein expression was then immediately visualized and quantified using an infrared imaging system (LI-COR Biosciences). Protein expression was normalized to cell number using the DRAQ5 and Sapphire700 stains. The activated Akt expression levels were normalized to total Akt expression.

Immunocytochemistry and microscopy

For protein expression and localization analysis of integrin $\alpha 5$ and $\beta 1$ in pAEC cultures, fluorescent immunocytochemistry and confocal microscopy was utilized. For imaging of cultured pAEC following wounding, pAEC of children with and without wheeze were cultured on $0.8-\mathrm{cm}$ round glass coverslips (precoated with FN, type I collagen, and BSA). Cultures were grown to confluence, scratch wounded with sterile p300 pipette tip, and - 24 hours after wounding - fixed using $4 \%$ (w/v) PFA for 20 minutes at room temperature and washed 3 times for 5 minutes per wash with $1 \times$ PBS. The cells were stored in $1 \times \mathrm{PBS}$ at $4^{\circ} \mathrm{C}$ until required. For immunocytochemical staining for integrin $\alpha 5$ and $\beta 1$ expression patterns, cultured pAEC were equilibrated to room temperature and incubated with Zuk's blocking buffer containing 10\% (v/v) FBS and 5\% (w/v) BSA in PBS for 1 hour at room temperature, as previously described (11). Following blocking, primary antibodies against human integrin subunits $\alpha 5$ (1:100; clone EPR7854, Abcam) and $\beta 1$ (1:1000; clone P5D2, Abcam) diluted in Zuk's blocking buffer were incubated for 1 hour at room temperature, followed by three 5 -minute washes with $1 \times$ PBS and incubation with secondary antibodies Alexa Fluor 568 goat anti-rabbit IgG (1:200; A-11036, Molecular Probes) and Alexa Fluor 488 goat anti-mouse IgG (1:200; A-32723, Molecular Probes) for 1 hour at room temperature. Finally, the cells were washed $3 \times$ for 5 minutes each with $1 \times$ PBS. Hoechst $33342(2.5 \mu \mathrm{g} / \mathrm{mL}$; Sigma-Aldrich) counterstain was used as a nuclear marker in all confocal fluorescence images. The coverslips were mounted onto glass slides with antifade fluorescence mounting media (DAKO), and edges were sealed and dried prior to imaging. Cultured pAEC were acquired using a Nikon A1 inverted confocal microscope, with a Nikon Plan Apo VC 60x Numerical Aperture (NA) 1.4 oil immersion objective and processed with Nikon Instrument Software (NIS) Elements-Advanced Research (AR) (v4.2.22) and ImageJ (NIH) software. Individual channels were captured sequentially, where a 405-nm laser was used for Hoechst 33342 with collection through a 450/50 
bandpass filter, AF488 excited using a $488 \mathrm{~nm}$ laser with collection through 525/50, and AF568 excited with a $561 \mathrm{~nm}$ laser with collection through a 585/50 bandpass filter. Z-stack images with step size of $0.5 \mu \mathrm{m}$ were collected with a pinhole of $35.8 \mu \mathrm{m}$ (1.2 AU for $488 \mathrm{~nm}$ laser), where the top and bottom of the stacks were determined visually.

\section{Leading edge cell isolation}

Submerged pAEC cultures were grown on 12-well plates (precoated with FN, type I collagen, and BSA) until $100 \%$ confluence was achieved. Once completely confluent, cell monolayer cultures were incubated in wounding media for 24 hours and subsequently scratch wounded with a sterile p300 pipette tip. Plates were then washed using wounding media to remove any cellular debris. Wounded cultures were incubated at $37^{\circ} \mathrm{C}$ over a 24 -hour period. Cells lining the leading edge of the wound were collected 24 hours after wounding. Leading edge cells were detached from the cell monolayer by scraping behind the first 3 rows of cells from the wound edge using a p200 Gilson pipette tip under stereomicroscopic guidance (modular stereomicroscope Leica MZ6, Leica Microsystems) and sterile conditions. Leading edge cells were pooled from 2 wells to collect sufficient cells and RNA for downstream analyses. Isolated cells were collected in BEBM and centrifuged at $700 \mathrm{~g}$ for 3 minutes at $4^{\circ} \mathrm{C}$ to form a cell pellet. Cells were lysed in QIAzol lysis buffer, snap frozen in liquid nitrogen, and stored at $-80^{\circ} \mathrm{C}$ until all samples were collected and extracted to minimize any potential multibatch variance.

\section{RNA extraction and RNA sequencing (RNA-Seq) analysis}

$R N A$ extraction and cDNA library preparation for RNA-Seq. Total RNA was extracted using QIAzol lysis buffer and the miRNeasy micro kit (QIAGEN) according to manufacturer's instructions. Following extraction, total RNA purity, yield, and integrity was determined via NanoDrop spectrophotometer, Qubit fluorometer, and Agilent 2100 Bioanalyzer, respectively. Samples containing at least $500 \mathrm{ng}$ of total RNA of high purity (A260/280 > 2.0) and quality $(\mathrm{RIN}>7.5)$ were processed for library preparation.

The KAPA Stranded mRNA-Seq kit (KAPABiosystems, Roche) was used for mRNA capture. RNA fragments were subsequently reverse transcribed into cDNA strands, which was followed by adapter ligation and library amplification for single-end read sequencing. The quality and quantity of all RNASeq libraries were assessed using Agilent Bioanalyzer and Qubit, respectively. RNA-Seq libraries were sequenced on the Illumina HiSeq 2500 platform at the UBC Sequencing Centre using the RAPID mode and generated $100 \mathrm{bp}$ single-end reads at a sequencing depth of $>3$ million reads per sample, followed by bcl2fastq conversion and demultiplexing. Sequencing quality of all fastq files was assessed based on GC content, average base quality and Illumina adapter contamination using MultiQC (version 1.5) (58). Raw reads are deposited under GSE117489 (GEO Accession, https://www.ncbi.nlm.nih.gov/geo/query/ acc.cgi? acc $=$ GSE117489) and PRJNA482321 (BioProject).

Data processing and differential gene expression analysis. A metadata table was constructed containing all clinical and sample-specific information. Subsequently, the single-end sequence reads were mapped to the reference genome (Homo sapiens GRCh38 v91, Ensembl) using STAR (version 2.6.0c). The count reads were then indexed to the metadata table using htseq-count (version 0.6.1p1). Alignment quality was assessed with MultiQC (version 1.5) (58). Sample variance was inspected with principal component analysis (PCA) and heatmap. To establish a signature gene expression profile from pAEC from children with or without wheeze, DESeq2 (version 1.20.0) was used to identify genes that had statistically significant differential expression (Benjamini-Hochberg-adjusted $P<0.050$, fold changes $|1.5|$ ) between "wheeze leading edge $24 \mathrm{~h}$ " and "non-wheeze leading edge $24 \mathrm{~h} . "$

Data interpretation. NetworkAnalyst $(59,60)$ and Ingenuity Pathway Analysis (IPA, Ingenuity Systems, QIAGEN Inc., Redwood City, California, USA) were used to facilitate systems-level analyses by enabling the integration, analysis, and visualization of gene expression data, in the context of molecular interaction networks and canonical pathways. Several statistical and analytical tools have been integrated into IPA, which were used to rapidly determine canonical pathways that were statistically overrepresented in the transcriptomic experiments of interest. Network analysis of expression changes were performed using NetworkAnalyst $(59,60)$, which is based on curated protein/protein interactions from InnateDB (61) - the largest database of interactions curated with respect to genes involved in host responses and provides a major advantage in permitting the creation of substantially enriched networks relevant to integrins, cell migration, and wound repair. 
IPA was also used to identify transcriptional regulators of integrin $\alpha 5$ and $\beta 1$ expression with upstream regulator analysis. upstream regulator analysis is a predictive causal analysis tool that identifies upstream molecules or networks of molecular drivers, including transcription factors and endogenous chemicals that may regulate the observed transcriptional differences in the data sets. Specifically, this analysis compares the overlap between known downstream targets of each transcriptional regulator with the DE gene lists, and is thus statistically predicted to identify molecules regulating the observed differential expression pattern. Also, IPA predicts whether the transcriptional regulators are activated or inhibited, based on known relationships between each regulator and the expression (upor downregulation) of the downstream targets within each data set. These predictions are reported as activation $\mathrm{Z}$ scores and are a second method for ranking predicted upstream transcriptional regulators after the adjusted $P$ values. Positive $\mathrm{Z}$ scores indicate activation, and negative $\mathrm{Z}$ scores indicate inhibition of the upstream regulator. Absolute activation $\mathrm{Z}$ scores $\geq 2$ and adjusted $P<0.050$ were considered significant, as recommended by IPA.

\section{Evaluation of published transcriptomic data sets}

Two published transcriptomic data sets (GSE103166 at https://www.ncbi.nlm.nih.gov/geo/query/ acc.cgi?acc=GSE103166 and https://www.synapse.org/\#!Synapse:syn9878922/files), were selected for validation of our upstream regulator analysis based on utilizing comparable ex vivo pAEC isolation protocols (i.e., nasal airway samples from children with and without wheeze, or nonasthmatic and asthmatic adults). Although these studies used different transcriptomic platforms - e.g., Affymetrix Human Gene 2.1 ST Array (39), Agilent Genome 4X44K array (16), Illumina HiSeq 1000 (17), and Illumina HiSeq 2500 (38) - as compared with Illumina HiSeq 2500 in our study, the cutoffs for differential gene expression analysis were the same $( \pm 1.5$-fold, adjusted $P<0.050)$. Differentially expressed gene lists comparing gene expression in ex vivo pAEC from asthmatic and nonasthmatic adults at baseline and after rhinoviral infection were investigated. Using prior knowledge of protein/protein interactions, minimum networks were generated with NetworkAnalyst, and pathway analysis (Reactome) was performed, where data sets were screened for overrepresentation of the PI3K/Akt signaling and integrin pathways. Weighted gene coexpression network analysis (WGCNA) was utilized to identify gene coexpression networks (modules) or similar function genes that associated with recurrence of respiratory wheeze in the pediatric data set $(39,62)$. Using WGCNA, gene expression was correlated with sample trait values (gene significance [GS]) and module membership (MM). Genes with high GS and high MM were considered to be significantly associated with recurrence and modules with possibly common biological processes.

\section{Statistics}

Statistical significance $(P<0.050)$ for comparisons between 2 groups were determined by Fisher's exact test for categorical variables and Mann-Whitney $U$ test for continuous variables (nonparametric). For multiple comparisons of compound treatments and disease phenotypes, nonparametric 2-way Kruskal-Wallis ANOVA with Dunn's multiple comparisons test were utilized. For the analysis of defective pAEC repair in a cohort of 119 children, data were fit into a generalized linear mixed model including respiratory wheeze (predictor variable), age, and sex (both fixed effects), and we took into account the variation in patient recruitment cohorts used in this analysis: iNOS and WAERP (random effects). For the subanalysis of defective pAEC repair in either pre-school-aged ( $\leq 5$ years) or school-aged ( $>5$ years old) children, data included wheeze (predictor variable), sex (fixed effect), and patient recruitment cohorts (random effect) and were fitted into a generalized linear mixed model. Experiments presented in Figures 1-6 and Supplemental Figures 1-7 were performed in technical duplicates and using at least 6 patients of each cohort per experiment, or as otherwise listed in figure legends.

\section{Study approval}

The study was approved by the Perth Children's Hospital, St. John of God Hospital, and The University of Western Australia's Human Ethics Committees, and written consent was obtained from each participant's legal guardian after being fully informed about the nature and purpose of the study. All experiments were performed in accordance with the Perth Children's Hospital, St. John of God Hospital, and the University of Western Australia committees' guidelines and regulations. 


\section{Author contributions}

TI optimized the in vitro wound repair protocols, conducted all experiments, analyzed and interpreted all data, prepared the manuscript for publication, and was involved in concept design. ENS, KML, KL, LWG, KMM, NCS, and STM all assisted with the human sample recruitment, sample processing, and cell culture establishment. AGB conducted the immunocytochemical staining and confocal microscopy. EKS recruited study participants. YVK assisted with statistical data analysis and presentation. PLS and IAL coordinated the pediatric wheeze longitudinal cohort, contributed cohort patient metadata, and critically revised the manuscript. TI, LC, EEG, and AHL were involved in the data analysis of the RNA-Seq data. FJL and SV performed the airway sampling and critically revised the manuscript. PJR, REWH, DAK, SMS, and AK were involved in the concept design and coordination of the study and critically revised the manuscript. $\mathrm{AK}$ and SMS contributed equally toward this manuscript and, thus, take senior author responsibility for the final content, interpretation, and integrity of the data.

\section{Acknowledgments}

This work was supported by grants from the National Health and Medical Research Council of Australia (NHMRC1048910). TI was supported by scholarships from the Australian Government, Centre for Cell Therapy and Regenerative Medicine, and Asthma Foundation of Western Australia. SMS is a NHMRC Practitioner Fellow. AK is a Rothwell Family Fellow. We would like to thank Anthony Bosco, Travis M. Blimkie, and Patricia Agudelo Romero for the technical expertise on transcriptomic data analysis and interpretation. Also, we would like to thank Lidija Turkovic for assistance with statistical data analysis in this manuscript. Special Acknowledgments are extended to Associate Professor Raelene Endersby for the generous donation of MK2206 and expertise on PI3K signaling and its experimental investigation. The authors acknowledge the facilities and the scientific and technical assistance of the Australian Microscopy and Microanalysis Research Facility at the Centre for Microscopy, Characterisation and Analysis (The University of Western Australia), a facility funded by the University, state, and commonwealth governments. We would like to thank the contribution and assistance of all the respiratory fellows, anesthetists, nurses, and hospital staff at Perth Children's Hospital and St. John of God. We would also like to thank the families and children on WAERP and the acute pediatric wheeze cohort, Mechanisms of Acute Viral Respiratory Infections in Children (MAVRIC), for their contributions to this study. See Supplemental Acknowledgments for consortia (WAERP \& AusREC) details.

Address correspondence to: Anthony Kicic, Telethon Kids Institute, Northern Entrance, Perth Children's Hospital, 15 Hospital Avenue, Nedlands, Western Australia, Australia, 6009. Phone: 618.96319.1799; Email: Anthony.Kicic@telethonkids.org.au.

1. Masoli M, Fabian D, Holt S, Beasley R, Global Initiative for Asthma (GINA) Program. The global burden of asthma: executive summary of the GINA Dissemination Committee report. Allergy. 2004;59(5):469-478.

2. Childhood Asthma Management Program Research Group, et al. Long-term effects of budesonide or nedocromil in children with asthma. N Engl J Med. 2000;343(15):1054-1063.

3. Bisgaard H, Hermansen MN, Loland L, Halkjaer LB, Buchvald F. Intermittent inhaled corticosteroids in infants with episodic wheezing. N Engl J Med. 2006;354(19):1998-2005.

4. Martinez FD. Inhaled corticosteroids and asthma prevention. Lancet. 2006;368(9537):708-710.

5. O’Byrne PM, Pedersen S, Lamm CJ, Tan WC, Busse WW, START Investigators Group. Severe exacerbations and decline in lung function in asthma. Am J Respir Crit Care Med. 2009;179(1):19-24.

6. O'Brian AL, Lemanske RF, Evans MD, Gangnon RE, Gern JE, Jackson DJ. Recurrent severe exacerbations in early life and reduced lung function at school age. J Allergy Clin Immunol. 2012;129(4):1162-1164.

7. van de Veen W, Akdis M. New Targets for Immune Modulation in Asthma. Curr Treat Options Allergy. 2014;1(2):171-185.

8. Ling KM, et al. Reduced transforming growth factor $\beta 1$ (TGF- $\beta 1$ ) in the repair of airway epithelial cells of children with asthma. Respirology. 2016;21(7):1219-1226

9. Barbato A, et al. Epithelial damage and angiogenesis in the airways of children with asthma. Am J Respir Crit Care Med. 2006;174(9):975-981.

10. Saglani S, et al. Ultrastructure of the reticular basement membrane in asthmatic adults, children and infants. Eur Respir J. 2006;28(3):505-512.

11. Kicic A, Sutanto EN, Stevens PT, Knight DA, Stick SM. Intrinsic biochemical and functional differences in bronchial epithelial cells of children with asthma. Am J Respir Crit Care Med. 2006;174(10):1110-1118.

12. Laitinen LA, Heino M, Laitinen A, Kava T, Haahtela T. Damage of the airway epithelium and bronchial reactivity in patients with asthma. Am Rev Respir Dis. 1985;131(4):599-606.

13. Puddicombe SM, et al. Involvement of the epidermal growth factor receptor in epithelial repair in asthma. FASEB J. 
2000;14(10):1362-1374.

14. Looi K, et al. Effects of human rhinovirus on epithelial barrier integrity and function in children with asthma. Clin Exp Allergy. 2018;48(5):513-524

15. Stevens PT, Kicic A, Sutanto EN, Knight DA, Stick SM. Dysregulated repair in asthmatic paediatric airway epithelial cells: the role of plasminogen activator inhibitor-1. Clin Exp Allergy. 2008;38(12):1901-1910.

16. McErlean $\mathrm{P}$, et al. Asthmatics with exacerbation during acute respiratory illness exhibit unique transcriptional signatures within the nasal mucosa. Genome Med. 2014;6(1):1.

17. Heymann PW, et al. Rhinovirus infection results in stronger and more persistent genomic dysregulation: Evidence for altered innate immune response in asthmatics at baseline, early in infection, and during convalescence. PLoS ONE. 2017;12(5):e0178096.

18. Redington AE, et al. Transforming growth factor-beta 1 in asthma. Measurement in bronchoalveolar lavage fluid. Am J Respir Crit Care Med. 1997;156(2 Pt 1):642-647.

19. Humbles AA, et al. A critical role for eosinophils in allergic airways remodeling. Science. 2004;305(5691):1776-1779.

20. Vignola AM, et al. Airway remodelling assessed by sputum and high-resolution computed tomography in asthma and COPD. Eur Respir J. 2004;24(6):910-917.

21. Holgate ST, Lackie P, Wilson S, Roche W, Davies D. Bronchial epithelium as a key regulator of airway allergen sensitization and remodeling in asthma. Am J Respir Crit Care Med. 2000;162(3 Pt 2):S113-S117.

22. Holgate ST, Arshad HS, Roberts GC, Howarth PH, Thurner P, Davies DE. A new look at the pathogenesis of asthma. Clin Sci. 2009;118(7):439-450.

23. Tsartsali L, et al. Development of the bronchial epithelial reticular basement membrane: relationship to epithelial height and age. Thorax. 2011;66(4):280-285.

24. Elliot JG, et al. Distribution of airway smooth muscle remodelling in asthma: relation to airway inflammation. Respirology. 2015;20(1):66-72.

25. James AL, et al. Airway basement membrane perimeter distensibility and airway smooth muscle area in asthma. J Appl Physiol. 2008;104(6):1703-1708.

26. Grainge CL, et al. Effect of bronchoconstriction on airway remodeling in asthma. N Engl J Med. 2011;364(21):2006-2015.

27. Kicic A, et al. Decreased fibronectin production significantly contributes to dysregulated repair of asthmatic epithelium. Am $J$ Respir Crit Care Med. 2010;181(9):889-898.

28. Lotz MM, Nusrat A, Madara JL, Ezzell R, Wewer UM, Mercurio AM. Intestinal epithelial restitution. Involvement of specific laminin isoforms and integrin laminin receptors in wound closure of a transformed model epithelium. Am J Pathol. 1997;150(2):747-760.

29. Nusrat A, Delp C, Madara JL. Intestinal epithelial restitution. Characterization of a cell culture model and mapping of cytoskeletal elements in migrating cells. J Clin Invest. 1992;89(5):1501-1511.

30. White SR, Dorscheid DR, Rabe KF, Wojcik KR, Hamann KJ. Role of very late adhesion integrins in mediating repair of human airway epithelial cell monolayers after mechanical injury. Am J Respir Cell Mol Biol. 1999;20(4):787-796.

31. Hérard AL, et al. Fibronectin and its alpha 5 beta 1-integrin receptor are involved in the wound-repair process of airway epithelium. Am J Physiol. 1996;271(5 Pt 1):L726-L733.

32. Kicic A, et al. Impaired airway epithelial cell responses from children with asthma to rhinoviral infection. Clin Exp Allergy. 2016;46(11):1441-1455.

33. Bain J, et al. The selectivity of protein kinase inhibitors: a further update. Biochem J. 2007;408(3):297-315

34. Liu Y, Shreder KR, Gai W, Corral S, Ferris DK, Rosenblum JS. Wortmannin, a widely used phosphoinositide 3-kinase inhibitor, also potently inhibits mammalian polo-like kinase. Chem Biol. 2005;12(1):99-107.

35. Hamdulay SS, et al. Celecoxib activates PI-3K/Akt and mitochondrial redox signaling to enhance heme oxygenase-1-mediated anti-inflammatory activity in vascular endothelium. Free Radic Biol Med. 2010;48(8):1013-1023.

36. Zhang PX, et al. Pharmacological modulation of the AKT/microRNA-199a-5p/CAV1 pathway ameliorates cystic fibrosis lung hyper-inflammation. Nat Commun. 2015;6:6221.

37. Han M, Rajput C, Ishikawa T, Jarman CR, Lee J, Hershenson MB. Small Animal Models of Respiratory Viral Infection Related to Asthma. Viruses. 2018;10(12):E682.

38. Pandey G, et al. A Nasal Brush-based Classifier of Asthma Identified by Machine Learning Analysis of Nasal RNA Sequence Data. Sci Rep. 2018;8(1):8826.

39. Khoo SK, et al. Upper Airway Cell Transcriptomics Identify a Major New Immunological Phenotype with Strong Clinical Correlates in Young Children with Acute Wheezing. J Immunol. 2019;202(6):1845-1858.

40. Nissen SE, et al. Cardiovascular Safety of Celecoxib, Naproxen, or Ibuprofen for Arthritis. N Engl J Med. 2016;375(26):2519-2529.

41. Simon RA. Adverse respiratory reactions to aspirin and nonsteroidal anti-inflammatory drugs. Curr Allergy Asthma Rep. 2004;4(1):17-24.

42. West PM, Fernández C. Safety of COX-2 inhibitors in asthma patients with aspirin hypersensitivity. Ann Pharmacother. 2003;37(10):1497-1501.

43. Celik G, et al. Tolerability of selective cyclooxygenase inhibitor, celecoxib, in patients with analgesic intolerance. $J$ Asthma 2005;42(2):127-131.

44. Sun YZ, Cai N, Liu NN. Celecoxib Down-Regulates the Hypoxia-Induced Expression of HIF-1 $\alpha$ and VEGF Through the PI3K/AKT Pathway in Retinal Pigment Epithelial Cells. Cell Physiol Biochem. 2017;44(4):1640-1650.

45. Liu B, Yan S, Qu L, Zhu J. Celecoxib enhances anticancer effect of cisplatin and induces anoikis in osteosarcoma via PI3K/Akt pathway. Cancer Cell Int. 2017;17:1.

46. Patel AR, Chougule MB, Patlolla R, Wang G, Singh M. Efficacy of aerosolized celecoxib encapsulated nanostructured lipid carrier in non-small cell lung cancer in combination with docetaxel. Pharm Res. 2013;30(5):1435-1446.

47. Shah PN, et al. Antimicrobial Activity of Ibuprofen against Cystic Fibrosis-Associated Gram-Negative Pathogens. Antimicrob Agents Chemother. 2018;62(3):e01574-17.

48. Martinovich KM, et al. Conditionally reprogrammed primary airway epithelial cells maintain morphology, lineage and disease 
specific functional characteristics. Sci Rep. 2017;7(1):17971.

49. Asher MI, et al. International Study of Asthma and Allergies in Childhood (ISAAC): rationale and methods. Eur Respir J. 1995;8(3):483-491.

50. Ferris BG. Epidemiology Standardization Project (American Thoracic Society). Am Rev Respir Dis. 1978;118(6 Pt 2):1-120.

51. Lane C, et al. Epithelial inducible nitric oxide synthase activity is the major determinant of nitric oxide concentration in exhaled breath. Thorax. 2004;59(9):757-760.

52. Lane C, Burgess S, Kicic A, Knight D, Stick S. The use of non-bronchoscopic brushings to study the paediatric airway. Respir Res. 2005:6:53.

53. Liu X, et al. ROCK inhibitor and feeder cells induce the conditional reprogramming of epithelial cells. Am J Pathol. 2012;180(2):599-607.

54. Sundaram GM, et al. 'See-saw' expression of microRNA-198 and FSTL1 from a single transcript in wound healing. Nature. 2013;495(7439):103-106.

55. Cordelieres F. Manual Tracking. NIH. http://rsb.info.nih.gov/ij/plugins/track/track.html. Updated June 15, 2005. Accessed March 16, 2020

56. Cory AH, Owen TC, Barltrop JA, Cory JG. Use of an aqueous soluble tetrazolium/formazan assay for cell growth assays in culture. Cancer Commun. 1991;3(7):207-212.

57. He JQ, et al. Selection of housekeeping genes for real-time PCR in atopic human bronchial epithelial cells. Eur Respir J. 2008;32(3):755-762.

58. Ewels P, Magnusson M, Lundin S, Käller M. MultiQC: summarize analysis results for multiple tools and samples in a single report. Bioinformatics. 2016;32(19):3047-3048.

59. Xia J, Gill EE, Hancock RE. NetworkAnalyst for statistical, visual and network-based meta-analysis of gene expression data. Nat Protoc. 2015;10(6):823-844.

60. Xia J, Benner MJ, Hancock RE. NetworkAnalyst--integrative approaches for protein-protein interaction network analysis and visual exploration. Nucleic Acids Res. 2014;42(Web Server issue):W167-W174.

61. Breuer K, et al. InnateDB: systems biology of innate immunity and beyond--recent updates and continuing curation. Nucleic Acids Res. 2013;41(Database issue):D1228-D1233.

62. Langfelder P, Horvath S. WGCNA: an R package for weighted correlation network analysis. BMC Bioinformatics. 2008;9:559. 